\title{
Luminosities and mass-loss rates of SMC and LMC AGB stars and red supergiants ${ }^{\star}, \star \star$
}

\author{
M. A. T. Groenewegen ${ }^{1}$, G. C. Sloan ${ }^{2}$, I. Soszyński ${ }^{3}$, and E. A. Petersen ${ }^{4,5}$
}

\author{
1 Koninklijke Sterrenwacht van België, Ringlaan 3, 1180 Brussels, Belgium \\ e-mail: marting@oma.be \\ 2 Cornell University, Astronomy Department, Ithaca, NY 14853-6801, USA \\ 3 Warsaw University Observatory, Al. Ujazdowskie 4, 00-478 Warszawa, Poland \\ 4 University of Nebraska, Department of Physics and Astronomy, Lincoln, NE 68588, USA \\ 5 NSF REU Research Assistant, Cornell University, Astronomy Department, Ithaca, NY 14853-6801, USA
}

Received 11 June 2009 / Accepted 20 August 2009

\section{ABSTRACT}

Context. Mass loss is one of the fundamental properties of Asymptotic Giant Branch (AGB) stars, and through the enrichment of the interstellar medium, AGB stars are key players in the life cycle of dust and gas in the universe. However, a quantitative understanding of the mass-loss process is still largely lacking, particularly its dependence on metallicity.

Aims. To investigate the relation between mass loss, luminosity and pulsation period for a large sample of evolved stars in the Small and Large Magellanic Cloud.

Methods. Dust radiative transfer models are presented for 101 carbon stars and 86 oxygen-rich evolved stars in the Magellanic Clouds for which 5-35 $\mu \mathrm{m}$ Spitzer IRS spectra are available. The spectra are complemented with available optical and infrared photometry to construct the spectral energy distribution. A minimisation procedure is used to fit luminosity, mass-loss rate and dust temperature at the inner radius. Different effective temperatures and dust content are also considered. Periods from the literature and from new OGLE-III data are compiled and derived.

Results. We derive (dust) mass-loss rates and luminosities for the entire sample. Based on luminosities, periods and amplitudes and colours, the O-rich stars are classified as foreground objects, AGB stars and Red Super Giants. For the O-rich stars silicates based on laboratory optical constants are compared to "astronomical silicates". Overall, the grain type by Volk \& Kwok (1988, ApJ, 331, 435) fits the data best. However, the fit based on laboratory optical constants for the grains can be improved by abandoning the smallparticle limit. The influence of grain size, core-mantle grains and porosity are explored. A computationally convenient method that seems to describe the observed properties in the $10 \mu \mathrm{m}$ window are a distribution of hollow spheres with a large vacuum fraction (typically 70\%), and grain size of about $1 \mu \mathrm{m}$.

Relations between mass-loss rates and luminosity and pulsation period are presented and compared to the predictions of evolutionary models, those by Vassiliadis \& Wood (1993, ApJ, 413, 641) and their adopted mass-loss recipe, and those based on a Reimers mass-loss law with a scaling of a factor of five. The Vassiliadis \& Wood models describe the data better, although there are also some deficiencies, in particular to the maximum adopted mass-loss rate. The derived mass-loss rates are compared to predictions by dynamical wind models and appear consistent with them at a level of a factor $2-4$. A better understanding requires the determination of the expansion velocity from future observations from ALMA.

The OGLE-III data reveal an O-rich star in the SMC with a period of 1749 days. Its absolute magnitude of $M_{\text {bol }}=-8.0$ makes it a good candidate for a super-AGB star.

Key words. stars: AGB and post-AGB - stars: mass loss - Magellanic Clouds

\section{Introduction}

Almost all stars with initial masses in the range $\sim 0.9-8 M_{\odot}$ will pass through the asymptotic giant branch (AGB) phase, which is the last stage of active nuclear burning before they become postAGB stars, planetary nebulae and finally white dwarfs. Slightly more massive stars will pass through the red supergiant (RSG) phase before they may end as supernovae. In both cases, massloss dominates the final evolutionary stages of the star.

\footnotetext{
* Complete Figs. 1-3 and 5-7 are only available in electronic form at http://www. aanda.org

$\star \star$ Complete Tables 1-4 are only available in electronic form at the CDS via anonymous ftp to cdsarc.u-strasbg.fr (130.79.128.5) or via

http://cdsweb.u-strasbg.fr/cgi-bin/qcat?J/A+A/506/1277
}

Although this is well-known and studied in detail in galactic sources with the advent of the Infrared Astronomical Satellite (IRAS) and the Infrared Space Observatory (ISO), uncertainties in distances lead to uncertainties in luminosities and massloss rates. Sources at known distances, as in Large and Small Magellanic Clouds (LMC and SMC), reduce this problem, and also allow one to study the effect of metallicity on the mass-loss rate.

In a previous paper, Groenewegen et al. (2007) modelled the spectral energy distribution (SED) and spectra taken with the Infrared Spectrograph (IRS; Houck et al. 2004) onboard the Spitzer Space Telescope (Werner et al. 2004) for a sample of 60 carbon (C) stars. They concluded that, assuming similar expansion velocities and dust-to-gas ratios as in Galactic stars, mass-loss rates versus luminosity or pulsation period scatter 
around the galactic relation for sources in both the LMC and $\mathrm{SMC}$ sources. In other words, there is no evidence that the massloss rate of $\mathrm{C}$ stars depends on metallicity. Recent theoretical work also suggests that lower metallicity does not necessarily imply smaller mass-loss rates for carbon stars (Mattsson et al. 2008; Wachter et al. 2008). The detection of dust forming around a C star in the Sculptor Dwarf Spheroidal galaxy (Sloan et al. 2009), with a metallicity of $[\mathrm{Z} / \mathrm{H}]=-1.3$, supports the theory observationally.

Sloan et al. (2008) compared Spitzer spectroscopy of C stars and oxygen-rich AGB stars and RSGs (hereafter referred to as $M$ stars). They found that while the carbon stars showed little dependence of mass loss on metallicity, the amount of dust produced by $\mathrm{M}$ stars declined in more metal-poor environments. The aim of the present paper is to extend the analysis by considering nearly $90 \mathrm{M}$ stars and enlarging the sample of C stars to over 100 .

Section 2 describes the sample of AGB stars and RSG with IRS spectra, the photometry to be fitted, and the derivation of pulsation periods. Section 3 describes the radiative transfer model and the properties of the dust species considered. Section 4 presents the results in the form of tables with the fitted parameters and figures comparing the models to the SEDs and IRS spectra. Section 5 discusses the results. In particular, we attempt to separate the O-rich stars into foreground, AGB stars and RSG. We discuss the influence of different assumptions on the shape and size of the grain on the fit to the IRS spectra. We also examine how the mass-loss rate depends on luminosity and period and compare our results to evolutionary models. Section 6 summarises the findings.

\section{The sample}

Several groups have obtained Spitzer IRS data of evolved stars in the LMC and SMC. In this paper we consider the currently publically available data from the following programmes: 200 (P.I. J. Houck), 3277 (P.I. M. Egan), 3426 (P.I. J. Kastner), 3505 (P.I. P. Wood), and 3591 (P.I. F. Kemper). The data in these programs are described by Sloan et al. (2008, program 200), Sloan et al. (2006, program 3277), Buchanan et al. (2006, program 3426), Zijlstra et al. (2006) and Lagadec et al. (2007) for program 3505, and Leisenring et al. (2008, program 3591). We have retrieved the spectra from these programs from the public archive and reduced them in a uniform manner, as described by Sloan et al. $(2006,2008)$. The reader should refer to these works for more details. Here, we outline how spectra are produced from the IRS data. All of the spectra were taken using the low-resolution modules on the IRS, Short-Low (SL) covering 5.2-14.3 $\mu \mathrm{m}$, and Long-Low (LL) covering 14.2-37.0 $\mu \mathrm{m}$. The standard low-resolution observation placed the source in two nod positions in each spectral aperture. The spectral images were differenced to remove background emission and cleaned to correct bad pixels. Spectra were extracted from the images using the tools available in SPICE, which is distributed by the Spitzer Science Center. Spectra from the separate nods were combined using a spike-rejection algorithm to remove features in one nod spectrum but not the other. Spectra from the separate apertures and modules were then combined, using multiplicative shifts to remove discontinuities between them and finally removing extraneous data from the ends of each spectral segment.

The five programs considered here did not exclusively observe AGB stars and RSGs. Targets were selected from these programs by examining the IRS spectra, collecting additional photometry (see below), consulting SIMBAD and the papers describing these programs, and considering our radiative transfer models (see Sect. 3). Excluded sources include those with very poor S/N IRS data, sources where the SED and spectrum did not match at all (indicating that the IRS peak-up was on a source other than the intended target), sources with a likely disk geometry (invalidating the spherically symmetric radiative transfer model used here), a post-AGB star (MSX SMC 029; Kraemer et al. 2006), two RCrB stars (MSX SMC 014, MSX SMC 155; Kraemer et al. 2005), O/Be-stars, and objects showing PAH emission ${ }^{1}$. The remaining sample includes the very interesting object WOH G 64, even though Ohnaka et al. (2008) have recently demonstrated with interferometric observations that the mid-IR visibility curves and the SED can be better modelled with a torus.

The sample under consideration consists of $101 \mathrm{C}$-stars and 86 M-stars. Tables 1 and 2 list basic information: some common names (as listed by SIMBAD), an OGLE-III identifier when this lightcurve is analysed and shown in Fig. 3, R.A. and declination in decimal degrees, the identifier used in figures and tables below, the adopted pulsation period, the (semi-)amplitude of the adopted pulsation period in the filter where the lightcurve was obtained, i.e. OGLE $I$, MACHO $B, R$, ASAS $V, I$, or in the $K$ band (Only the first entries are shown for guidance; both tables are available in their complete form at the CDS). The stars are listed in order of their luminosity, from brightest to faintest as determined below (and assuming these sources are in the LMC and SMC).

Many of the periods quoted in Tables 1 and 2 come from the published literature, but in some cases publically available data were re-analysed if the quoted period did not seem to match the lightcurve or if published periods did not agree with each other. Figures 1 and 2 show our fits to publically availably $\mathrm{ASAS}^{2}$ (Pojmanski 2002) and MACHO data ${ }^{3}$. In addition, we used unpublished data from the OGLE-III survey (Udalski et al. 2008), and when available, combined this with OGLE-II data. In these cases, Fig. 3 shows the observed data and the fitted lightcurve. Tables 1 and 2 list the adopted pulsation period, but many of the stars for which we (re-)analysed the light curve show additional periods. These are listed in Appendix A.

For all stars additional broad-band photometry ranging from the optical to the mid-IR was collected from the literature, primarily using VizieR ${ }^{4}$ and the NASA/IPAC Infrared Science Archive $^{5}$, using the coordinates listed in Tables 1 and 2. In particular, we considered

- In the optical: $U B V I$ data from Zaritsky et al. (2002, 2004) for the Magellanic Clouds (MCs). $U B V R$ data from Massey (2002) for the MCs, BVRI data from Oestreicher et al. (1997)

\footnotetext{
1 In detail, the following sources from these programs are not considered. From program 200: WBP 219, WBP 29, WBP51, NGC 371 LE8, NGC 381 LE31, NGC 371 LE28, WBP 104, WBP 116, HV 5810, HV 5680, IRAS 04530-6916; from program 3277: MSX SMC 014, 029, 079, 125, 155, 180; from program 3426: MSX LMC 559, 1306, 1794, 217, 222, 22, 764, 836, 889, 894, 934, 773, 769, 890; from program 3505: NGC 419 LE 35, GM 106, RAW 1559, ISO-MCMS J005149.4-731315 (iso00518), IRAS 05328-6827; from program 3591: MSX LMC 616, LHA 120-N 89, LHA 120-N 77d, BSDL 2894, BSDL 126, [O96] D010b-262, HD 38489, NGC 1978 WBT2665, NGC 1948 WBT2215, HD 269924, MSX LMC 795, MSX LMC 1786, [L63] 31, MSX LMC 906, [SL63] 482, IRAS 04514-6913, MSX LMC 610.

${ }^{2}$ http://www.astrouw.edu.pl/asas/

3 See http://wwwmacho.anu.edu.au/

${ }^{4}$ http://vizier.u-strasbg.fr/viz-bin/VizieR

5 http://irsa.ipac.caltech.edu/
} 
Table 1. The C-star sample: identifiers and pulsation periods for the first few entries.

\begin{tabular}{|c|c|c|c|c|c|c|}
\hline Names & RA & Declination & Identifier & Period & Ref. & Ampl. (Filter) \\
\hline IRAS 04496-6958, MSX LMC 1130 & 72.327000 & -69.887361 & iras04496 & 723,741 & 1,18 & $0.44(K)$ \\
\hline IRAS 05278-6942, MSX LMC 635 & 81.850458 & -69.662472 & $\operatorname{iras} 05278$ & 980 & 9 & $1.20(K)$ \\
\hline IRAS 00554-7351, [GB98] S16 & 14.266458 & -73.587389 & iras00554 & 720 & 8 & $0.72(K)$ \\
\hline MSX LMC 1298, LMC134.1 40407 & 74.133958 & -68.880833 & msxlmc1298 & 683 & $\mathrm{pp}$ & $1.12(I)$ \\
\hline MSX LMC 775, LMC166.2 19640, MACHO 8.8541.68 & 83.234083 & -68.213528 & msxlmc775 & 2067, 2170 & $\mathrm{pp}, \mathrm{pp}$ & $.84(I), 0.62(R)$ \\
\hline
\end{tabular}

References: $\mathrm{pp}=$ present paper $; 1=$ Whitelock et al. (2003); $2=$ Groenewegen (2004); $3=$ Nishida et al. (2000); $4=$ Raimondo et al. (2005); $5=$ Reid et al. (1988); $6=$ Sloan et al. (2006); 7 = Wood (1998); 8 = Dodion (2003); $9=$ Wood et al. (2008, in prep.); $10=$ Groenewegen et al. (2007), 11 = Hughes (1989); 12 = Whitelock et al. (1994), 13 = Wood et al. (1992); 14 = Lloyd Evans (1985); $15=$ Wood et al. (1983, periods actually from Payne-Gaposhkin, 1971); 16 = Cioni et al. (2003); 17 = Sloan et al. (2008); 18 = Fraser et al. (2008); $19=$ Pojmanski (2002, ASAS data in general).

Table 2. The M-star sample: identifiers and pulsation periods for the first few entries.

\begin{tabular}{|c|c|c|c|c|c|c|}
\hline Names & RA & Declination & Identifier & Period & Ref. & Ampl. (Filter) \\
\hline MSX LMC 1677, IRAS 06013-6505 & 90.365833 & -65.089750 & msxlmc1677 & 348,340 & $\mathrm{pp}, 19$ & $2.1(I)$ \\
\hline 71832, MSX LMC 1687, IRAS 06045-6722 & 91.106208 & -67.388444 & 832 & $514,52.7$ & pp, 19 & $0.16(V)$ \\
\hline JMC 1686 & 91.699125 & -66.803472 & msxlmc 1686 & & & \\
\hline RAS 05169-7350, MSX LMC 412 & 78.921917 & -73.787139 & rsmen & 304 & 12 & $0.53(K)$ \\
\hline WOH G 17, MSX LMC 1150 & 69.848708 & -73.184111 & wohg17 & & & \\
\hline HD 269788, MSX LMC 778 & 83.723625 & -68.777639 & hd269788 & 15.9 & $\mathrm{pp}$ & $0.007(I)$ \\
\hline
\end{tabular}

for RSG in the LMC, OGLE BVI data from Udalski et al. (1998), VRI data from Wood et al. (1983, hereafter WBF).

- In the near-infrared: DENIS IJK data from Cioni et al. (2000) and the third data release (The DENIS consortium 2005), the all-sky $J H K$ release of 2MASS (Skrutskie et al. 2006), and the extended mission 6x long-exposure release, $J H K$ data from the IRSF survey (Kato et al. 2007), SAAO JHKL data from Whitelock et al. (1989, 2003), and CASPIR $J H K L$ data specifically taken for the IRS observations (Sloan et al. 2006, 2008; Groenewegen et al. 2007), and from Wood et al. (1992), Wood (1998).

- In the mid-IR: IRAS data from the Point Source Catalog, and the Faint Source Catalog (Moshir et al. 1989), IRAC 3.6,4.5,5.8,8.0 and MIPS $24 \mu \mathrm{m}$ data from the SAGE cata$\log$ (Meixner et al. 2006, first epoch data) and $\mathrm{S}^{3} \mathrm{MC}$ catalog (Bolatto et al. 2007).

The literature considered is not exhaustive but does include all recent survey data available in the near- and mid-IR, where these stars emit most of their energy.

\section{The model}

The models are based on the dust radiative transfer (RT) algorithm of Groenewegen (1993; also see Groenewegen 1995), which was developed to handle non $-r^{-2}$ density distributions in spherically symmetric dust shells. The algorithm simultaneously solves the radiative transfer equation and the thermal balance equation for the dust.

The models for $\mathrm{C}$ stars begin with the stellar atmosphere models by Loidl et al. (2001; available for $T_{\text {eff }}=2650,2800$, $3000,3200,3600 \mathrm{~K}$ ), while for M stars the M0-M10 model atmospheres of Fluks et al. (1994) are used. These range from 3850 to $2500 \mathrm{~K}$. Strictly speaking, the models are valid for giants at solar metallicities, but we have applied them to Magellanic supergiants and AGB stars. MARCS models ${ }^{6}$ are not yet

\footnotetext{
${ }^{6}$ http://marcs.astro.uu.se/
}

available for abundances typical of AGB stars (i.e. with nonsolar $\mathrm{C} / \mathrm{O}$ ratios).

Our models assume that the dust around $\mathrm{C}$ stars is a combination of amorphous carbon (AMC) and silicon carbide (SiC), with optical constants from, respectively, Rouleau \& Martin (1991; the AC1 species), $\alpha$-SiC from Pégourié (1988), and $\beta$-SiC from Borghesi et al. (1985), taking into account the matrix correction factors (see footnote in Groenewegen 1995). These choices are based on the practical fact that these two types of dust fit actual observations. We are aware of the discussion by, e.g., Pitman et al. (2008) about inadequacies in the derivation of optical constants in the literature, and that $\beta$-SiC is probably the primary carrier of the $11.3 \mu \mathrm{m}$ feature in $\mathrm{C}$ stars, rather than $\alpha$-SiC, in agreement with meteoritic data (see the discussion by Speck et al. 2009). However, SiC features are known that clearly peak shortward of $11.3 \mu \mathrm{m}$, and these are better fitted with the constants of Borghesi et al. Section 5.4.1 discusses this point further.

Speck et al. also proposed that graphite rather then amorphous carbon dominates the dust, at least in the C-stars with extreme mass-loss rates they considered. Only one set of optical constants seems to have been published for graphite, those by Draine \& Lee (1984), as used by e.g. Volk et al. (1992), and Speck et al. Calculating the absorption coefficients for spherical grains using these optical constants results in a broad shoulder $\sim 40 \mu \mathrm{m}$, as is evident already in Fig. $4 \mathrm{~b}$ in Draine \& Lee. This is not observed in C-stars, and is the reason why e.g. Martin \& Rogers (1987) already dismissed graphite in favour of AMC. Independently, graphite is expected to form at temperatures as high as $1800 \mathrm{~K}$. As we will show below, when the condensation temperature is left as a free parameter it will typically be of order $1000 \mathrm{~K}$, which is consistent with the condensation temperature of AMC.

Many C stars show a broad feature around $30 \mu \mathrm{m}$ which is believed to arise from $\mathrm{MgS}$ dust (Goebel \& Moseley 1985). Hony et al. (2002) showed that the absorption coefficients depend strongly on the shape of the grains and that if $\mathrm{MgS}$ is the carrier, a continuous distribution of ellipsoids (CDE) is required. In addition they find that the temperature of the $\mathrm{MgS}$ 

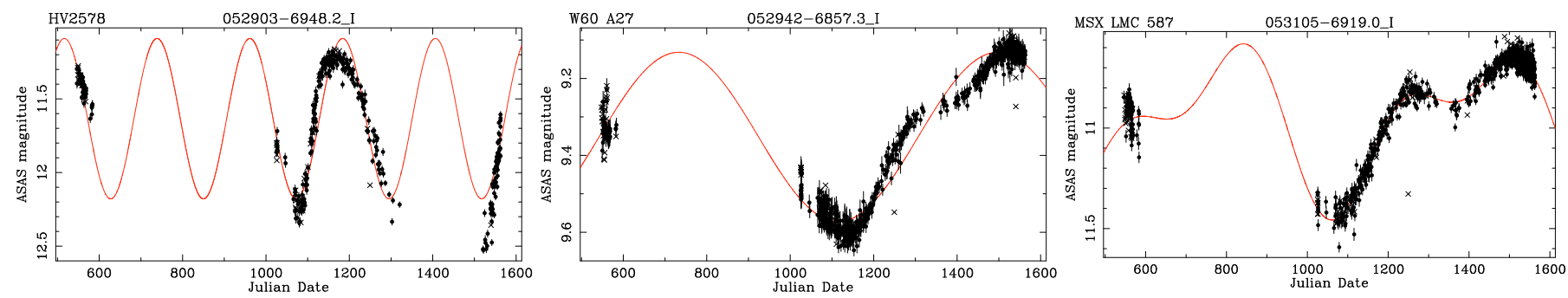

Fig. 1. Sample lightcurves and fits to ASAS data. The identifier used in the present paper and the ASAS identifier are listed on top of the plot. The complete figure is available in the electronic edition. Julian Date plotted is JD-2 450000.
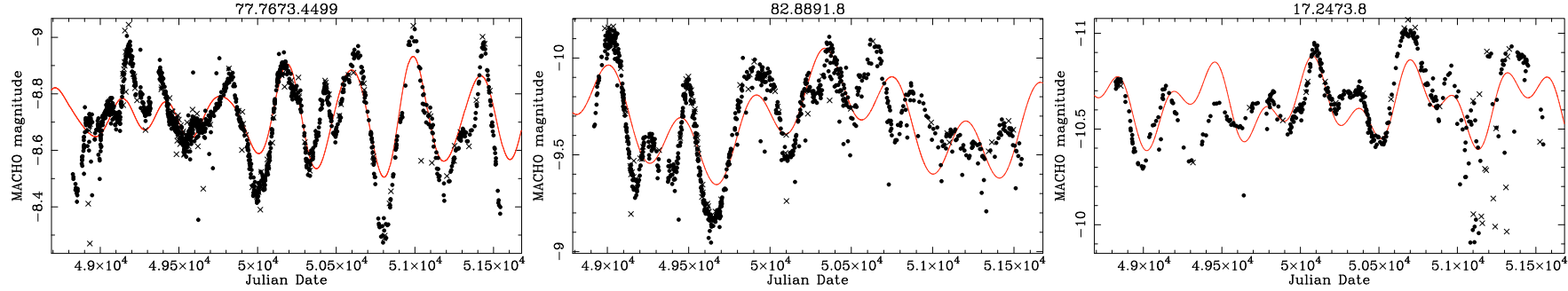

Fig. 2. Sample lightcurves and fits to MACHO data. MACHO identifiers are listed on top of the plot, and are cross-referenced in Tables 1 and 2. The complete figure is available in the electronic edition. Julian Date plotted is JD-2 400000.
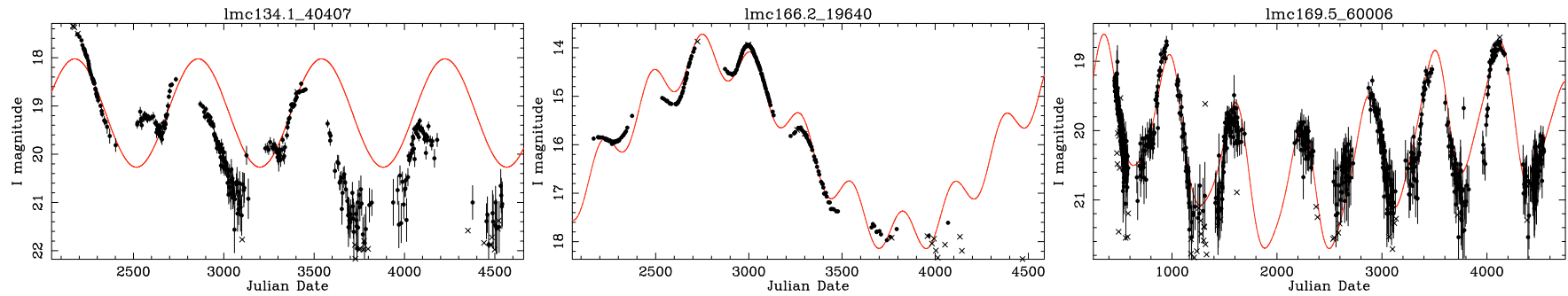

Fig. 3. Sample lightcurves and fits to OGLE-III data (and OGLE-II data when available). OGLE-III identifiers are listed on top of the plot, and are cross-referenced in Tables 1 and 2. The complete figure is available in the electronic edition. Julian Date plotted is JD-2 450000.

grains is very low, $100-400 \mathrm{~K}$ typically, and is thus unrelated to the warm dust close to the star which is primarily responsible for the infrared excess that we are modelling. Consequently, we exclude this wavelength region and $\mathrm{MgS}$ from our initial models. IRS observations confirm that $\mathrm{MgS}$ is associated with cool dust temperatures, leading Zijlstra et al. (2006) to argue that the $\mathrm{MgS}$ condenses onto existing grain surfaces. Leisenring et al. (2008) noted that the apparent SiC strength decreases as MgS increases, and they suggested that the $\mathrm{MgS}$ coating hides the $\mathrm{SiC}$ emission feature. In this scenario, our models would give only a lower limit to the fraction of the $\mathrm{SiC}$ in the dust. While we do not model the $\mathrm{MgS}$, the reader should keep in mind that its presence may mask spectral features at shorter wavelengths. Section 5.4.2 discusses the $30 \mu \mathrm{m}$ feature further.

For the M stars, several types of "astronomical silicates" are available, as well as combinations of optical constants taken from laboratory data. For "astronomical silicates" we used the absorption coefficients of Volk \& Kwok (1988, hereafter VK, scaled down by a factor of 5 to agree with most other silicates), Draine \& Lee (1984, hereafter DL), "warm" silicates from Suh (1999), Ossenkopf et al. (1992, hereafter OHM), David \& Pegourie (1995, hereafter DP), and combinations of DP with aluminium oxide ( $\mathrm{AlOx}$; amorphous porous $\mathrm{Al}_{2} \mathrm{O}_{3}$ ), with optical constants from Begemann et al. (1997), as used to model the SEDs of $\mathrm{M}$ stars in the Galactic Bulge (Blommaert et al. 2006).
We constructed "laboratory silicates" using mixtures of olivine $\left(\mathrm{Mg}_{0.8} \mathrm{Fe}_{1.2} \mathrm{SiO}_{4}\right.$, from Dorschner et al. 1995) and $\mathrm{AlOx}$ and metallic iron (Ordal et al. 1988). Harwit et al. (2001) and Kemper et al. (2002) have advocated the use of metallic iron to increase the opacity in the near-IR region, and the present models confirm this need. We used discrete combinations with 3, 4 or $5 \% \mathrm{Fe}$, and $0,10,20,30$ or $40 \% \mathrm{AlOx}$, with olivine accounting for the remainder.

Other dust components have been identified in the spectra of M-stars, e.g. spinel or corundum (Posch et al. 1999; Sloan et al. 1996), but these are only minor components and remain controversial (e.g., Sloan et al. 2003; Heras \& Hony 2005; Depew et al. 2006). The aim of the present paper is to globally fit the SED as opposed to fitting the details of the IRS spectra of the stars.

For the dust mixtures in both $\mathrm{C}$ and $\mathrm{M}$ stars, the extinction coefficients have been calculated in the small-particle limit, and $\left(Q_{\lambda} / a\right)$ is calculated from adding in proportion the extinction coefficients of the individual species. In other words, the grains are treated separately, but all have the same temperature. The standard model does not consider core-mantle grains, and the composition of the grains is independent of distance to the star. See Sect. 5.3 for further discussion.

Distances of $50 \mathrm{kpc}$ to the $\mathrm{LMC}$ and $61 \mathrm{kpc}$ to the SMC are adopted. The models have been corrected for a typical $A_{\mathrm{V}}=$ 0.15 for all stars. The exact value is of little importance as this corresponds to $\lesssim 0.02 \mathrm{mag}$ of reddening in the near-IR. For all 


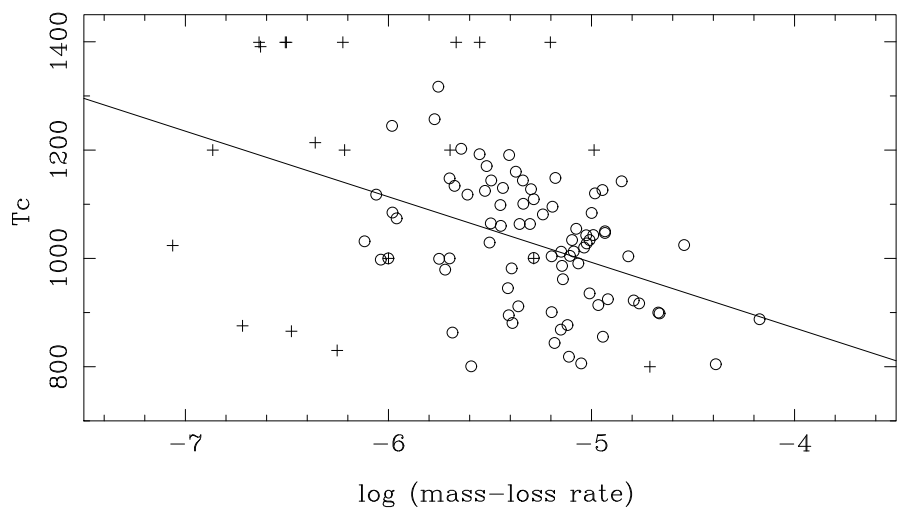

Fig. 4. Dust temperature at the inner radius versus mass-loss rate for $\mathrm{C}$ stars. Plus-signs indicate stars excluded from the fit (the solid line, Eq. (1)).

stars an expansion velocity, $v_{\mathrm{exp}}$, of $10 \mathrm{~km} \mathrm{~s}^{-1}$ and a dust-to-gas ratio of 0.005 have been adopted.

We fitted the models to the SEDs in the following manner. The RT model was included as a subroutine in a minimisation code using the the MRQMIN routine (using the Levenberg-Marquardt method from Press et al. 1992). Parameters that were fitted in the minimisation process include the mass-loss rate $(\dot{M})$, luminosity, and the dust temperature at the inner radius $\left(T_{\mathrm{c}}\right)$, although we sometimes fixed $\dot{M}$ and/or $T_{\mathrm{c}}$. The output of a model is an SED, and this is folded with the relevant filter response curves to produce magnitudes to compare to the observations (see Groenewegen 2006).

Typically, for a fixed effective temperature the model was minimised for the different grain types, for both fitted and fixed $T_{\mathrm{c}}$. For the grain type with the lowest $\chi^{2}$ error, the model was run for different model atmospheres. This is reasonable because the IRS spectrum largely determines the best-fitting model atmosphere, while the effective temperature is largely determined from the optical and near-IR broad-band photometry. When fixed, $T_{\mathrm{c}}$ was set at temperatures typically expected, i.e. $900,1000,1100,1200 \mathrm{~K}$. For C stars it has been suggested (e.g. Groenewegen 1995) that $T_{\mathrm{c}}$ decreases with optical depth. This effect has been investigated by first allowing $T_{\mathrm{c}}$ to vary. Excluding mass-loss rates below $6 \times 10^{-7} M_{\odot} \mathrm{yr}^{-1}$ where $T_{\mathrm{c}}$ and $\dot{M}$ are not simultaneously fitted well, and stars where the fit converged to temperatures above $1400 \mathrm{~K}$ or below $800 \mathrm{~K}$, a linear relation gives

$T_{\mathrm{c}}=(-121 \pm 29) \log \dot{M}+(386 \pm 156)$

based on 82 stars, with a mean of $1030 \mathrm{~K}$, see Fig. 4. In subsequent model runs, $T_{\mathrm{c}}$ was then also fixed to the value given by Eq. (1). For M stars such a dependence has never been suggested and has not been investigated.

For some C-stars it also turned out that lowering the outer radius of the dust shell markedly improved the fit. Groenewegen et al. (2007) discussed two cases previously, and Sloan et al. (2009) found this necessary for a C star in the Sculptor Dwarf. The default outer radius (as a multiple of the inner radius) is typically of order 10000 and is determined by considering a dust temperature at the outer radius of $20 \mathrm{~K}$. For stars where a smaller outer radius seemed necessary, new models were run decreasing the outer radius by a factor of 3 each time until the value of $\chi^{2}$ rose again. For none of the $\mathrm{M}$ stars a smaller outer radius was necessary, but for a non-negligible fraction of the C stars (20\%), it was. Although not explicitly tested, the same net effect (less flux at longer wavelengths) can be achieved by a steeper density law than the $r^{-2}$ assumed in the RT model.

The quality of the fit is based on a $\chi^{2}$ analysis

$\chi^{2}=\sum_{i=1}^{i=n}\left(m_{\mathrm{obs}}(i)-m_{\text {pred }}(i)\right)^{2} /\left(\sigma_{\mathrm{m}_{\mathrm{obs}}(\mathrm{i})}\right)^{2}$,

with $m$ the observed or predicted magnitude (for the broadband photometry) or flux (for the spectrum) with error bar $\sigma_{\mathrm{m}_{\mathrm{obs}}}$ and $n$ is the total number of measurements. Also the reduced $\chi^{2}$ is defined:

$\chi_{\mathrm{r}}^{2}=\frac{\chi^{2}}{(n-p)}$

with $p$ the number of free parameters, and the quantity

$\mathrm{BIC}=\chi^{2}+(p+1) \ln (n)$

This is based on the Bayesian information criterion (Schwarz 1978) and measures whether an increase in the number of free parameters and the resulting lower $\chi^{2}$ is actually significant. In this way it is possible, for example, to compare models with fitted and fixed $T_{\mathrm{c}}$, by comparing the values of BIC.

In Eq. (2) broadband data and spectral data are included in one formalism. However it is not evident how to weigh the typically 10-40 available photometric data points with the $\sim 350$ spectral data points. For a few stars, where the fit to the SED alone already provided a good match to the IRS spectrum both in absolute flux and spectral shape the following experiment was done. The IRS spectrum covers the 5 to $38 \mu \mathrm{m}$ region, and broadband photometry available in this region (IRAC 5.8 and $8.0 \mu \mathrm{m}$, MSX A, MIPS $24 \mu \mathrm{m}$, and IRAS 12 and $25 \mu \mathrm{m}$ bands) has a typical resolution of order 4-6. At such a resolution, 10-13 such filters fit within the $5-37 \mu \mathrm{m}$ region covered by the IRS. In the experiment we added, effectively duplicated, 10-13 of the available IRAC, MSX, MIPS, and IRAS, data points and calculated the resulting reduced $\chi^{2}$. Then the original photometric dataset was fitted including the IRS spectrum, and the uncertainties of the spectral data were scaled so as to produce the same reduced $\chi^{2}$. We examined four stars in this manner, found the scaling factors to be in the range 3-8, and concluded that the uncertainties of the IRS spectral data should be scaled by a factor of 5 .

We masked those portions of the IRS spectra with poor $\mathrm{S} / \mathrm{N}$ or those affected by background subtraction problems and did not include them in the minimisation procedure. In addition, regions where strong molecular features dominate that are not included in the simple $\mathrm{C}$-star model atmospheres are also excluded for the $\mathrm{C}$ stars, i.e. the regions 4.3-6.2 $\mu \mathrm{m}\left(\mathrm{CO}+\mathrm{C}_{3}\right.$, see e.g. Jørgensen et al. 2000), 6.6-8.5 $\mu \mathrm{m}$ and 13.5-13.9 $\mu \mathrm{m}\left(\mathrm{C}_{2} \mathrm{H}_{2}+\right.$ HCN band, see e.g. Matsuura et al. 2006), and 22.0-38.0 $\mu \mathrm{m}$ (the region of the $\mathrm{MgS}$ dust feature, which is not considered).

\section{Results}

Tables 3 and 4 lists the parameters of the models which best fit the observed data. (Only the first entries are shown for guidance. Both tables are fully available at the CDS). For the M stars, the first line lists the best overall fit, while the second lists the best fit with an "astronomical silicate" if the overall best fit is for a "laboratory silicate", and vice versa. Listed are the identifier, effective temperature or spectral type, dust type, luminosity, massloss rate, whether $\dot{M}$ was fitted (1) or fixed (0), $T_{\mathrm{c}}$, whether $T_{\mathrm{c}}$ was fitted or fixed, outer radius (in units of inner radius), dust optical depth in the $V$-band, optical depth at $11.3 \mu \mathrm{m}$ (C-stars), 
Table 3. Fit results of the C-star sample for the first few entries.

\begin{tabular}{rrrrrrrrrrr}
\hline \hline Identifier & $\begin{array}{r}T_{\text {eff }} \\
(\mathrm{K})\end{array}$ & $\begin{array}{rrrrrr}\text { grain } \\
\text { type }\end{array}$ & $\begin{array}{r}L \\
\left(L_{\odot}\right)\end{array}$ & $\begin{array}{r}\dot{M} \\
\left(M_{\odot} \mathrm{yr}^{-1}\right)\end{array}$ & fit? & $\begin{array}{r}T_{\mathrm{c}} \\
(\mathrm{K})\end{array}$ & fit? & $R_{\text {out }}$ & $\tau_{0.5}$ & $\tau_{11.3}$ \\
\hline iras04496 & 2800 & sic0 & 35690 & $5.18 \mathrm{e}-06$ & 1 & 1000 & 0 & 10000.0 & 4.646 & 0.145 \\
iras05278 & 3600 & sic0 & 30617 & $2.85 \mathrm{e}-05$ & 1 & 1055 & 0 & 6000.0 & 22.645 & 0.704 \\
iras00554 & 3600 & sic4 & 27127 & $1.42 \mathrm{e}-05$ & 1 & 1204 & 0 & 10000.0 & 16.465 & 0.752 \\
msxlmc1298 & 3600 & sic4 & 24506 & $1.15 \mathrm{e}-05$ & 1 & 1142 & 0 & 41.2 & 12.977 & 0.593 \\
msxlmc775 & 3200 & bsic2 & 20776 & $5.59 \mathrm{e}-06$ & 1 & 1082 & 1 & 4.6 & 7.170 & 0.284 \\
\hline
\end{tabular}

sic refers to $\alpha$-SiC from Pégourié (1988), bsic to $\beta$-SiC from Borghesi et al. (1985). The number refers to the percentage of SiC. The rest is amorphous carbon from Rouleau \& Martin (1991; also see Sect. 3).

Table 4. Fit results of the M-star sample for the first few entries.

\begin{tabular}{rrrrrrrrrrr}
\hline \hline Name & $\begin{array}{r}\text { Spectral } \\
\text { type (K) }\end{array}$ & $\begin{array}{r}\text { grain } \\
\text { type }\end{array}$ & $\begin{array}{r}L \\
\left(L_{\odot}\right)\end{array}$ & $\begin{array}{r}\dot{M} \\
\left(M_{\odot} \mathrm{yr}^{-1}\right)\end{array}$ & fit? & $\begin{array}{r}T_{\mathrm{c}} \\
(\mathrm{K})\end{array}$ & fit? & $R_{\text {out }}$ & $\tau_{0.5}$ & $\tau_{10}$ \\
\hline msxlmc1677 & $\mathrm{m} 8$ & $\mathrm{VK}$ & 2773703 & $2.63 \mathrm{e}-06$ & 1 & 802 & 1 & 10000.0 & 0.249 & 0.056 \\
msxlmc1677 & $\mathrm{m} 5$ & sil75alox20fe5 & 3194712 & $2.06 \mathrm{e}-06$ & 1 & 918 & 1 & 10000.0 & 0.106 & 0.034 \\
hd271832 & $\mathrm{m} 6$ & $\mathrm{VK}$ & 1787262 & $2.00 \mathrm{e}-08$ & 0 & 900 & 0 & 10000.0 & 0.003 & 0.001 \\
hd271832 & $\mathrm{m} 6$ & sil65alox30fe5 & 1791278 & $1.60 \mathrm{e}-08$ & 0 & 900 & 0 & 10000.0 & 0.001 & 0.000 \\
msxlmc1686 & $\mathrm{m} 10$ & DP 0.8 alox 0.2 & 1215022 & $9.42 \mathrm{e}-07$ & 1 & 1000 & 0 & 10000.0 & 0.134 & 0.032 \\
msxlmc1686 & $\mathrm{m} 10$ & si155alox40fe5 & 1235338 & $6.02 \mathrm{e}-07$ & 1 & 900 & 0 & 10000.0 & 0.056 & 0.018 \\
rsmen & $\mathrm{m} 9$ & $\mathrm{VK}$ & 724206 & $5.23 \mathrm{e}-07$ & 1 & 1000 & 0 & 10000.0 & 0.156 & 0.035 \\
rsmen & $\mathrm{m} 9$ & sil65alox30fe5 & 747279 & $5.74 \mathrm{e}-07$ & 1 & 900 & 0 & 10000.0 & 0.073 & 0.024 \\
\hline
\end{tabular}

Grain type refer to: VK = Volk \& Kwok (1988), DL = Draine \& Lee (1984), Suh = Suh (1999), OHM = Ossenkopf et al. (1992), DP = David \& Pegourie (1995), and combinations of DP with Aluminium Oxide (AlOx). The types marked silXaloxYfeZ, refer to X-percent olivine, Y-percent AlOx, and Z-percent metallic iron (see Sect. 3 for details).

or the peak of the silicate feature. (This depends on the type of silicate used, but is near $10 \mu \mathrm{m}$ )

Figures 5-7 in the electronic edition show the best fits, for the M stars again for both "astronomical silicates" and "laboratory silicates". The top panel shows the observed SED and IRS spectrum and the fitted model on an absolute scale, while the bottom panel shows the IRS spectrum, scaled to a quasi-continuum point based on the average flux in the $6.4-6.5 \mu \mathrm{m}$ region.

The fitting routine also provides uncertainties for the massloss rate, dust temperature at the inner radius, and luminosity. These are typically small, of order $1 \%$. The true errors are larger, and can be estimated from a comparison of model runs with different parameters. They are typically $10 \%$ in luminosity, $25 \%$ in mass-loss rate and $50 \mathrm{~K}$ in $T_{\mathrm{c}}$.

\section{Discussion}

\subsection{Foreground objects and the division between AGB stars and supergiants}

The brightest $\mathrm{M}$ stars in the sample have luminosities in excess of one million solar luminosities if they are indeed in the MCs. The present section tries to identify likely foreground (FG) objects. In addition the separation between RSGs and (oxygenrich) AGB stars is of interest. WBF separated RSGs and AGB Stars based on (1) the location of a source in a diagram plotting $M_{\mathrm{bol}}$ versus period, and (2) the amplitude of pulsation, with AGB stars showing larger amplitudes. Smith et al. (1995) used a similar diagram, and they also considering the presence or absence in the atmosphere of lithium, which indicates that the star has experienced hot bottom burning (HBB) and is thus at the upper end of the mass range for stars on the AGB.

Figure 8 shows this diagram, using different symbols for different $I$-band amplitudes (see caption). Stars without (known) period are plotted as crosses at negative period. Amplitudes in
$B, V, R$, and $K$ have been multiplied by, respectively, $0.14,0.18$, 0.66 , and 2.3 to estimate the amplitude in $I$. These ratios are based on values actually determined for the stars in our sample. Colour is another useful criterion, as it indicates the presence of circumstellar material. In the electronic edition, [3.6]-[8.0] colours are coded by different colours (see caption).

A check of the literature indicates two obvious FG objects. RS Men has a radial velocity of $140 \mathrm{~km} \mathrm{~s}^{-1}$, far lower then the $250-300 \mathrm{~km} \mathrm{~s}^{-1}$ typical of LMC objects. HD 269788 is listed as having a significant proper motion, and has a spectral type of K4 III. None of the other stars have spectral types, radial velocities, parallaxes or proper motion that would suggest a foreground nature.

HD 271832 is another likely FG object. The object is plotted at its period (514 days) which shows the largest amplitude $(0.16$ in $V)$. This may not be its pulsation period but possibly related to the LSP (long secondary period) phenomenon observed in AGB stars (see e.g. Soszyński 2007, and references therein). The object also shows a period of 52.7 days with an amplitude of $0.06 \mathrm{mag}$. This period and amplitude suggest that the object is a probable early-type giant in the FG.

Early-type FG stars are expected to have essentially no massloss rate (hereafter MLR) and higher temperatures than AGB stars. HD 269788 and HD 271832 have a fitted optical depth at $10 \mu \mathrm{m}$ of $\leq 0.001$, and a [3.6]-[8.0] colour $\leq 0.09$. Three other stars fulfill both criteria, and are also classified as FG: W60 D29, MSX LMC 1212 and HV 11366. The last object has a period of 183 days (derived from MACHO data), but a small amplitude. WBF reported a spectral type of MS and a period of 366 days for HV 11366, and based on this period Sloan et al. (2008) assumed it was a member of the SMC. The period quoted by WBF comes from Payne-Gaposhkin (1971). Either the shorter period was missed, or the star has switched from fundamental to overtone mode. 

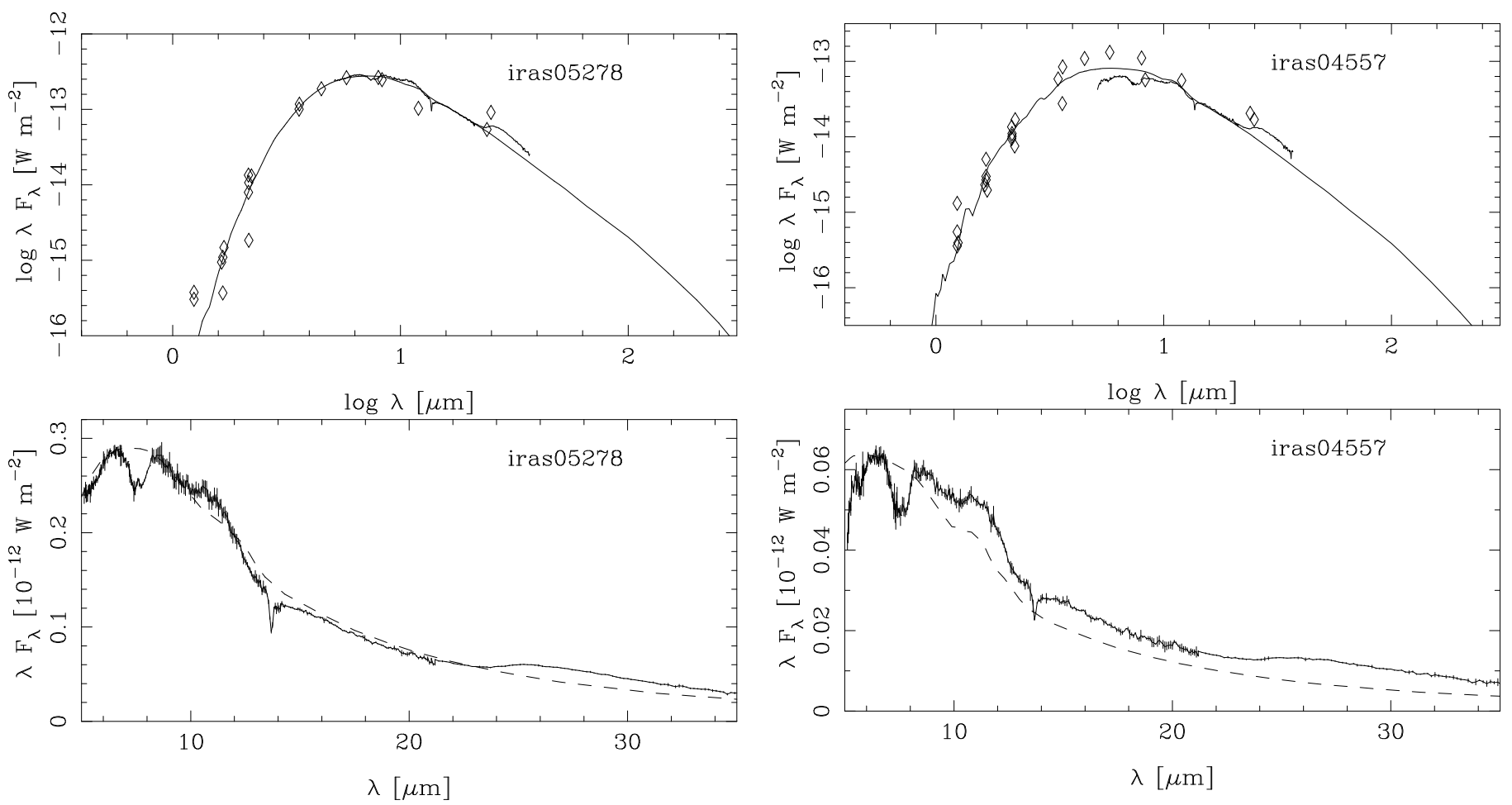

Fig. 5. Fits to the SEDs and IRS spectra of C-stars. The complete figure is available in the electronic edition.
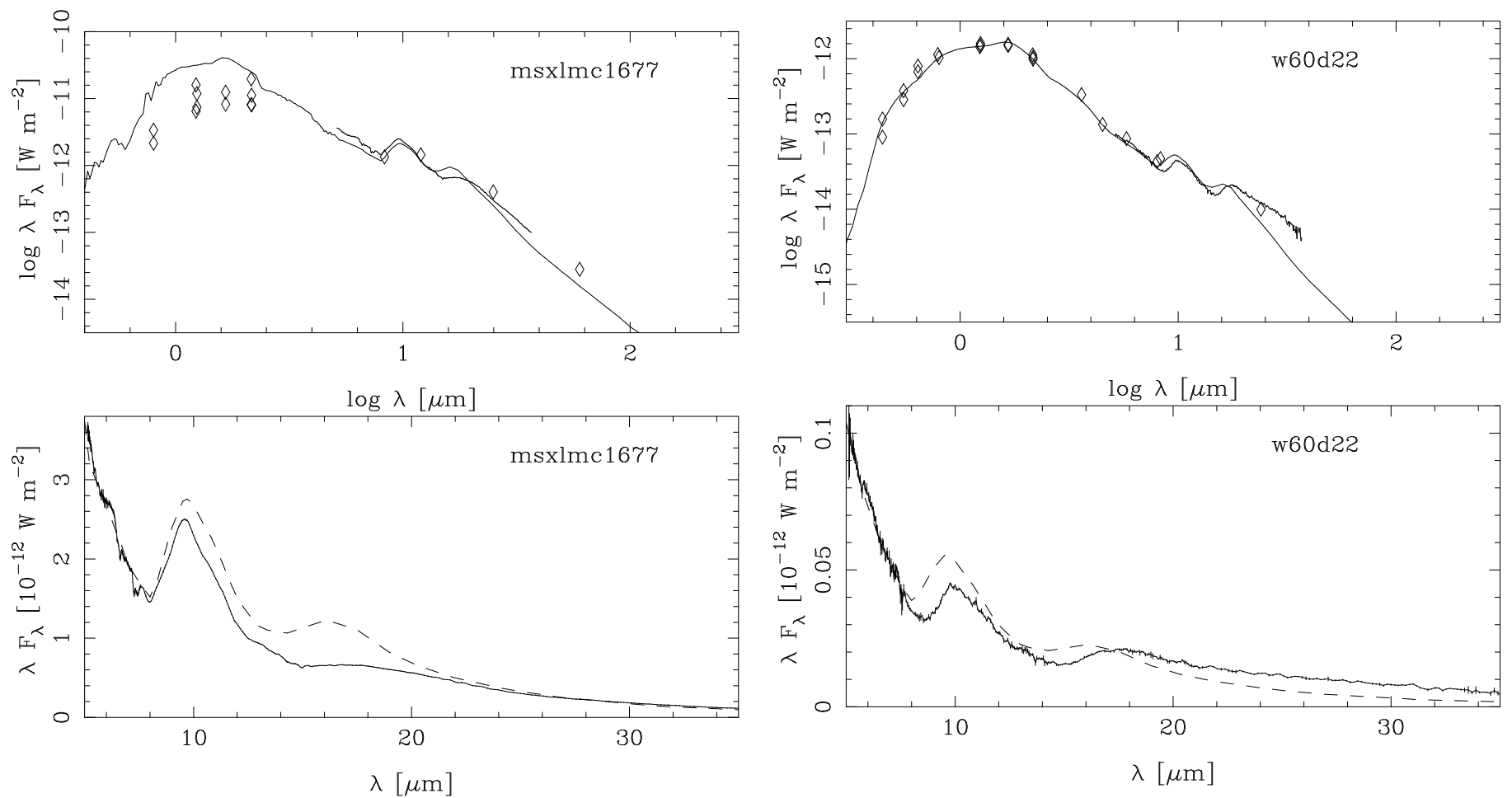

Fig. 6. Fits to the SEDs and IRS spectra of M-stars using "laboratory silicates". The complete figure is available in the electronic edition.

Buchanan et al. (2006) identified RS Men as a FG object because it is a Mira variable and its luminosity is not consistent with the Mira period-luminosity relation (Feast et al. 1989) if it were at the distance of the LMC, corroborating the FG nature suggested by its radial velocity. Buchanan et al. also used the $P L$ relation to identify MSX LMC 1677 and MSX LMC 1686 as FG Mira variables. If MSX LMC 1686 were in the LMC, its luminosity would exceed that of a very bright RSG $(L \approx 1.2$ million $L_{\odot}$ ). Similarly, WOH G 17 would have have a luminosity of $600000 L_{\odot}$, and thus we suspect that it is also FG. The circumstellar reddening and the [3.6]-[8.0] colours of MSX LMC 1686 and WOH G 17 are consistent with this suspicion. 

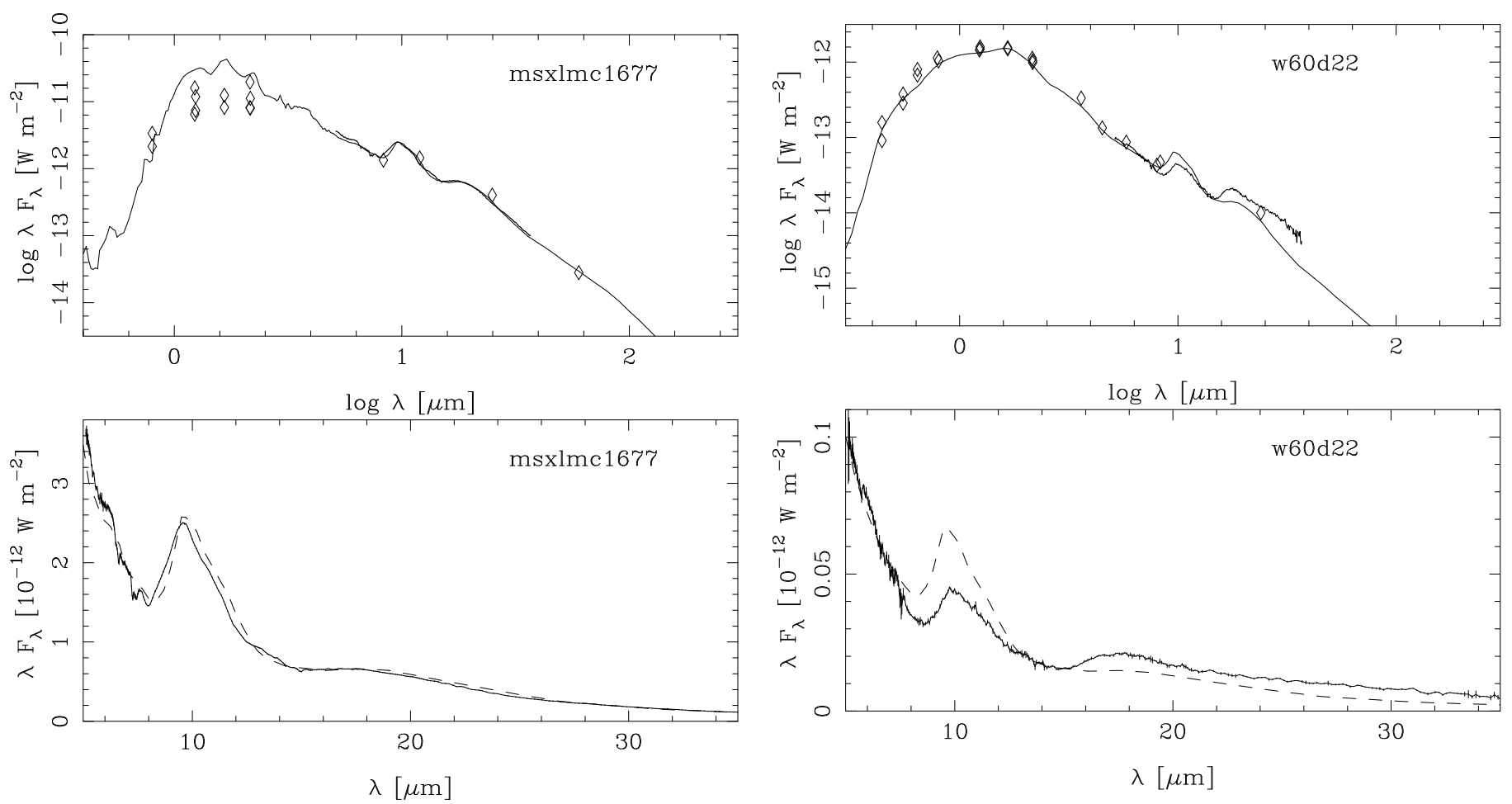

Fig. 7. Fits to the SEDs and IRS spectra of M-stars using "astronomical silicates". The complete figure is available in the electronic edition.

MSX LMC 946 is a SR with a period of 112 days. If it were in the LMC, it would also be too bright (with $L \approx 300000 L_{\odot}$ ). We believe it also is a FG star.

Both WBF and Smith et al. (1995) consider the maximum luminosity of an AGB star to be $M_{\mathrm{bol}}=-7.1$. This limit is based on the maximum possible core mass of $1.4 M_{\odot}$ before core He ignition and the core-mass luminosity relation of Paczyński (1970) or Wood \& Zarro (1981). Other limits have also been suggested. Wagenhuber \& Groenewegen (1998) give a limit of $L=16400,31800$ and $119000 L_{\odot}$ for $M_{\mathrm{c}}=0.8,1.0$ and $1.4 M_{\odot}$ or $M_{\mathrm{bol}}=-8.0$, respectively (although none of the full evolutionary calculations on which all of these core-mass luminosity relations are based have actually evolved a star to the Chandrasekhar limit). Poelarends et al. (2008) in their recent study of super-AGB stars examined a $9 M_{\odot}$ star. Their model had a core mass of $1.17 M_{\odot}$ after the second dredge-up at the start of the first thermal pulse, and it reached $\log L=5.07$ ( $\left.L=118000 L_{\odot}, M_{\text {bol }}=-8.0\right)$ at the twelfth pulse shortly after. These limits are consistent with the bolometric magnitude of the longest-period variable in the sample, MSX SMC 055, which has a period of 1749 days and an $I$-band amplitude of 0.81 . Among the stars in the sample, this object is the most likely candidate for a super-AGB star.

Stars (with or without period) brighter than $M_{\mathrm{bol}}=-8.0$ are classified as RSG. All of these turn out to have amplitudes lower than that expected for Mira variability. Therefore all stars with amplitudes smaller than 0.45 and in between the solid and dashed line in Fig. 8 are classified as RSG, and all remaining stars with amplitudes larger than 0.45 are classified as AGB stars.

Four stars with a known period have uncertain classifications. MSX LMC 1318 and HV 11223 are slightly fainter than the lower limit used by WBF, but have blue colours and small amplitudes and are tentatively classified as RSGs. MSX SMC 134 has a small amplitude but reasonable red colours and is suspected to be an AGB star. Similarly, WBP 77 has an amplitude close to that of a Mira variable and is treated as an AGB star.

Of the remaining stars without period, MSX LMC 807 is fainter than $M_{\mathrm{bol}}=-6.4$, has a red colour, [3.6]-[8.0] $>4$, and is classified as an AGB star. The remainder are brighter than $M_{\mathrm{bol}}=$ -7.8 , have blue colours, [3.6]-[8.0] $<0.1$, and are classified as RSG.

We have separated RSGs and AGB stars primarily to study the mass-loss process in these two classes of objects (see Sect. 5.5). However, the conclusions drawn there are not sensitive to the uncertainties in classification described here.

The brightest $\mathrm{C}$ star in the sample has a luminosity of $35000 L_{\odot}$, or $M_{\text {bol }}=-6.6$. The faintest has $4100 L_{\odot}$, or $M_{\text {bol }}=$ -4.3. The brightest $\mathrm{C}$ star in the SMC is the third brightest overall. The largest MLR is $7.1 \times 10^{-5} M_{\odot} \mathrm{yr}^{-1}$, for the seventh most luminous $\mathrm{C}$ star. Although generally speaking, a lower luminosity implies a smaller MLR, there are some exceptions. The fourth smallest MLR in the sample, $1.8 \times 10^{-7} M_{\odot} \mathrm{yr}^{-1}$, is for CV 78, with the thirteenth highest luminosity of $16000 L_{\odot}$.

Figure 9 shows the Hertzsprung-Russell diagram with evolutionary tracks of Bertelli et al. (2008), extended to unpublished tracks of more massive stars (Girardi, priv. comm.) for $Z=0.008$. For the intermediate-mass stars the evolution is stopped at the beginning of the AGB, which is why the tracks do not pass through the cloud of points marking the $\mathrm{O}$ - and C-rich AGB stars. Most of the RSGs are consistent with the 10-20 $M_{\odot}$ tracks (cf. the discussion on the revised spectral type effective temperature calibration in Levesque et al. 2005, 2006). The coolest RSG is MSX LMC 891 for which we assign a spectral type of M7 (3129 K).

\subsection{Colour-magnitude and colour-colour diagrams}

Figure 10 shows two colour-magnitude diagrams based on IRAC and NIR colours. The background of stars plotted as dots are 


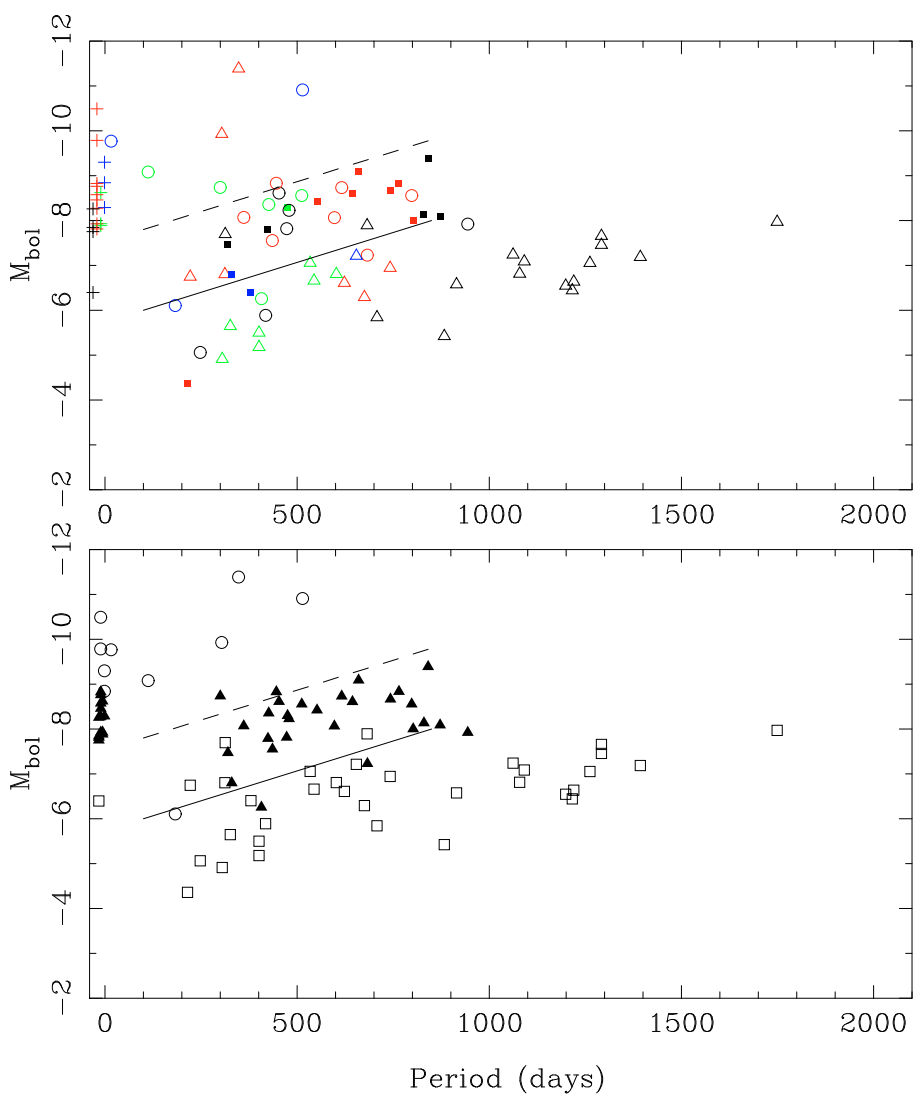

Fig. 8. Top panel. Bolometric magnitude versus pulsation period for the M stars. Stars without period are plotted as plus signs at negative periods. The full line indicates the lower luminosity limit for RSGs by WBF, and the dashed line is 1.8 mag brighter. Top panel. I-band semiamplitudes larger than $0.45 \mathrm{mag}$ are denoted by triangles. Amplitudes between 0.2 and 0.45 by filled squares. Amplitudes smaller then 0.2 by circles. In the electronic edition, [3.6]-[8.0] colours redder than 1.2 are shown in black, between 0.5 and 1.2 in red, between 0.15 and 0.5 in green, and less than 0.15 in blue. Bottom panel. As top panel, but the objects are identified as foreground objects (open circles), RSG (filled triangles), and AGB stars (open squares) based on the discussion in Sect. 5.1.

taken from a random subset of $\mathrm{S}^{3} \mathrm{MC}$ and SAGE data (and having errors in the magnitudes of less than $0.1 \mathrm{mag}$ ). The photometry plotted for the sample is the synthetic photometry calculated for the best fit model, and not the observed magnitudes.

Matsuura et al. (2009), Vijh et al. (2009), and others have recently published colour-magnitude diagrams with IRAC and/or NIR colours showing regions where particular types of objects can be found or overplotting stars with known spectral types. Our results agree with these previous works.

Figure 11 shows two colour-colour diagrams, in particular those that Kastner et al. (2008) indicate are effective in distinguishing $\mathrm{M}$ from $\mathrm{C}$ stars. We confirm this, in particular for the [5.8]-[8.0] vs. [8]-[24] diagram.

Figure 12 shows the bolometric correction (BC) at $3.6 \mu \mathrm{m}$ versus [3.6]-[8.0] colour, and at $K$ versus $J-K$ colour for the synthetic colours (also see WBF and Whitelock et al. 2003, for BCs involving $K$ and other colours). Relations like those presented here make it is possible to estimate bolometric magnitudes with an estimated uncertainty of about $0.2 \mathrm{mag}$, which might be sufficient for many applications. Such an estimate could even serve as a starting point for more detailed modelling.

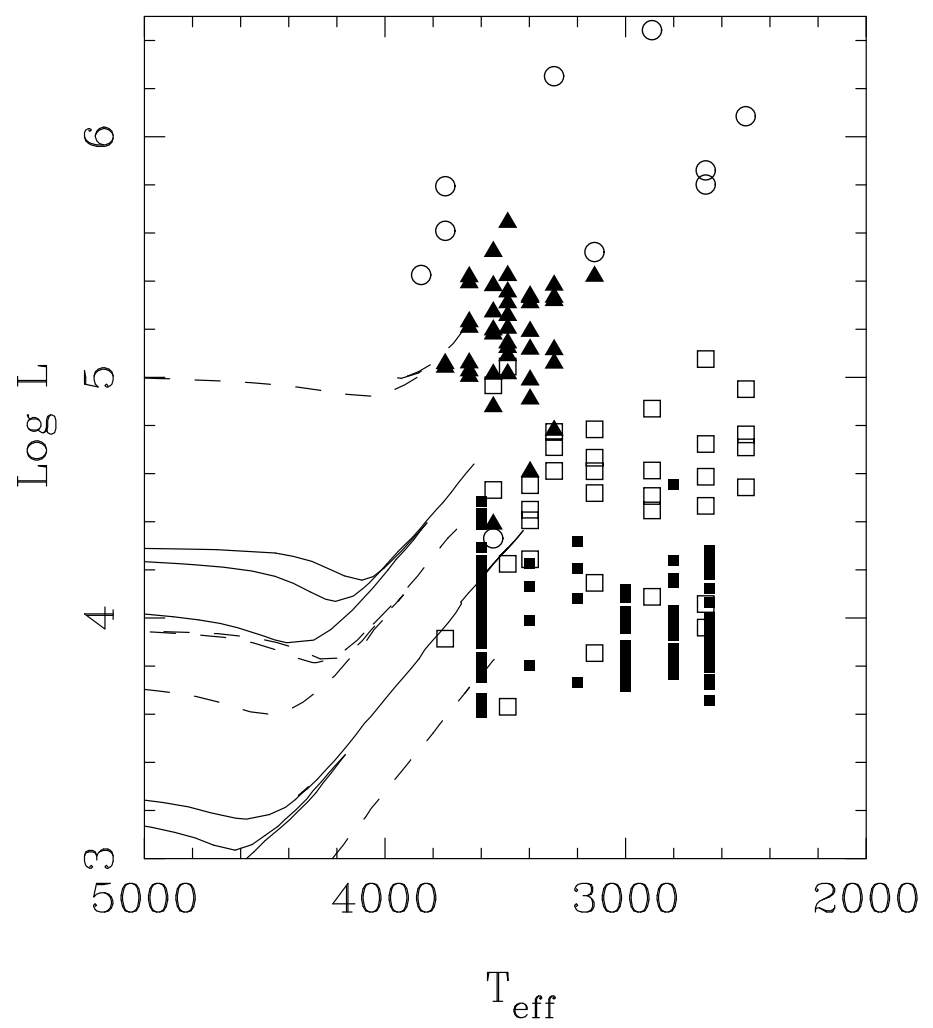

Fig. 9. The Hertzsprung-Russell diagram. C-stars are plotted as filled squares, M-type AGB stars as open squares, RSGs as filled triangles, and foreground objects as open circles. Lines indicate evolutionary tracks by Bertelli et al. (2008) for 20, 10, 8, 5, and $3 M_{\odot}$ (top to bottom) for $Z=0.008$.

\subsection{Dust in the M-stars}

The primary aim of the present paper is to derive luminosities and MLRs by fitting models to the broad-band data, as opposed to fitting the IRS spectra in detail. Nonetheless, some interesting remarks can and should be made.

We have considered two families of oxygen-rich dust. The first is the "astronomical silicates". These are derived empirically from observed spectroscopic data, generally galactic AGB stars. The second family is based on the optical constants measured in the laboratory, denoted here as "laboratory silicates". Figures 6 and 7 show the best fitting models determined independently for the two families.

Of the $86 \mathrm{M}$ stars, the MLR is fitted in 75 objects, and in 57 of those cases, an astronomical silicate provides the best overall fit. In most of those cases (70\%), the best fit comes from the "astronomical silicate" produced by Volk \& Kwok (1988), which is based on data from the Low-Resolution Spectrograph and photometry at $12,25,60$, and $100 \mu \mathrm{m}$ from IRAS of oxygen-rich AGB stars.

An inspection of Figs. 6 and 7 illustrates, however, that the best fitting models often give a far from perfect match to the data. In the astronomical silicates the $18 \mu \mathrm{m}$ feature is often too weak w.r.t. the $10 \mu \mathrm{m}$ feature, and broader than observed, Additionally, the $10 \mu \mathrm{m}$ peak is often too strong. The laboratory silicates produce the opposite problem: the $18 \mu \mathrm{m}$ feature is often too strong compared to the $10 \mu \mathrm{m}$ feature and peaks at too short of a wavelength. The $10 \mu \mathrm{m}$ feature peaks at slightly too short of a wavelength as well. In the 19 cases where the laboratory silicate provided the best fit, the model with the maximum considered value of 5\% metallic iron worked best in 17 cases. 

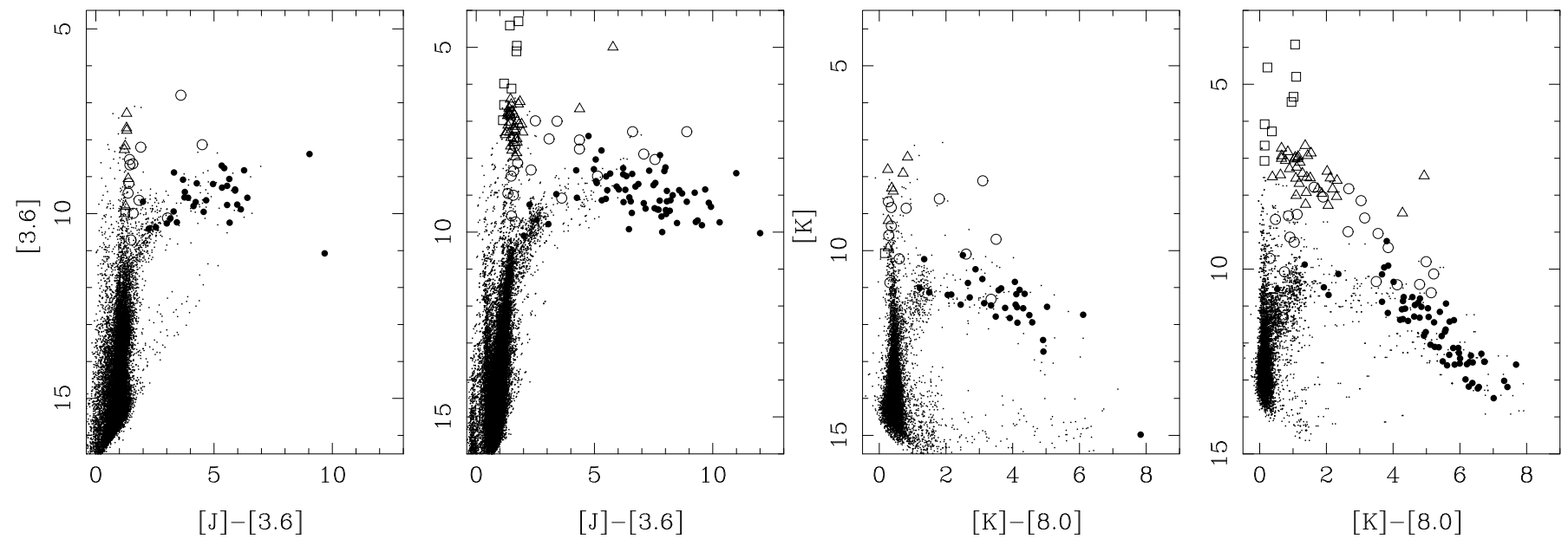

Fig. 10. Colour-magnitude diagrams from two combinations of colours for the SMC (left-hand panel) and LMC (right-hand panel). Dots are stars picked randomly from the $\mathrm{S}^{3} \mathrm{MC}$ and SAGE surveys. M stars are plotted as squares (foreground objects), triangles (supergiants), and circles (AGB stars). C stars are plotted as filled circles.
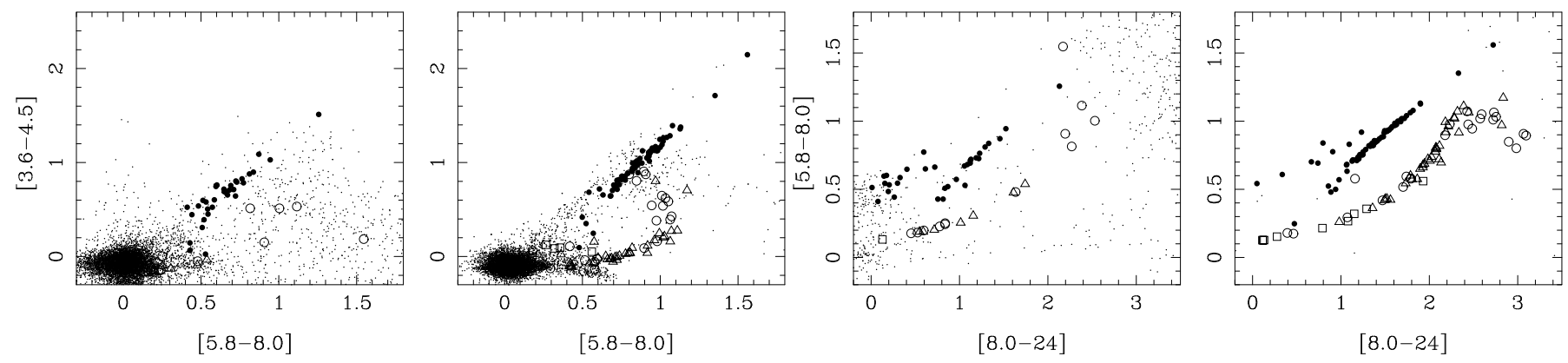

Fig. 11. Colour-colour diagrams for two combinations of colours for the SMC (left-hand panel) and LMC (right-hand panel). Dots are from the $\mathrm{S}^{3} \mathrm{MC}$ and SAGE survey. M stars are plotted as squares (foreground objects), triangles (supergiants), and circles (AGB stars). C stars are plotted as filled circles.

Kemper et al. (2002) used $4 \%$ in their study of a Galactic OH/IR star. The use of metallic iron is linked to the fact that laboratory silicates like olivine provide too little opacity in the near-IR, a notion that goes back to the introduction of the term "dirty silicates" by Jones \& Merrill (1976).

The opacity of the laboratory silicates has been calculated for small particles and for olivine and aluminium oxide for singlesized spherical grains. (For iron we followed the procedure of Kemper et al. to use a CDE.) The opacity of the mixture was calculated by adding the absorption coefficients of the components in proportion. The true composition and shape of the dust particles is immensely more complex than adopted here. One could consider the effects of ellipsoidal grains, CDE distributions, core-mantle grains, multi-layered grains, or even fluffy aggregates. It is likely that shape and size distribution and composition will all vary with distance from the central star, and may even vary with the phase of the pulsation cycle. We will briefly address some of these effects below, by comparing different models to observations of HV 12793. This source has an SED which is very well fitted, an optically thin shell, and is best fit with a mixture of laboratory silicates with 5\% iron and $95 \%$ olivine. This mixture is typical for our best-fitting laboratory silicate models.

Figure 13 shows the effect of different shapes, calculated for small particles, that have analytical solutions for the absorption properties (see Min et al. 2003): CDE, continuous distribution of spheroids (CDS), and a distribution of hollow spheres (DHS). In the last case, the optical properties are averaged over a uniform distribution in volume fraction between 0 and $f_{\max }$ of a vacuum core, where the material volume is kept constant. While CDE and CDS models probe the effect of deviations from homogeneous spherical grains, DHS models probe the effect of porosity. As Min et al. showed, the simple core-mantle approach in DHS is essentially equivalent to the general case of random vacuum inclusions according to effective medium theory (EMT). In the models, $T_{\mathrm{c}}$ was fixed and luminosity and MLR were fitted for grains with 3, 4 and 5\% iron. It turns out that CDE, CDS and DHS models show very similar results (with CDS models producing the best fits, formally). In all cases the best-fitting models have 5\% iron. Figure 13 shows the best fit with small spherical grains and with a CDS (both with $5 \%$ iron). Using a CDS (or CDE or DHS) improves the fit to the features at 10 and $18 \mu \mathrm{m}$.

Figure 14 shows the effect of different grain size, for a DHS with $f_{\max }=0$ (homogeneous spheres). It shows that grain size also has an effect on the fit to the $10-18 \mu \mathrm{m}$ region and that grains with a size around $1 \mu \mathrm{m}$ fit reasonably well.

Figure 15 shows the effect of porosity, with $f_{\max }=0.4,0.7$ and 0.9 for a grain size of $1.2 \mu \mathrm{m}$. Min et al. $(2005,2007)$ show that a DHS with $f_{\max }=0.7$ provides a good fit to the shape of the interstellar silicate feature at $10 \mu \mathrm{m}$, while with a value of 0.4 they can reproduce the polarisation properties of quartz measured in laboratory data. The effect of increasing the porosity on the spectra does not appear to be very large, mostly increasing the opacity at long wavelengths, so the value of $f_{\max }$ is not well constrained from the present data. Luminosity and MLR were minimised in all of these calculations, and the best-fitting values 

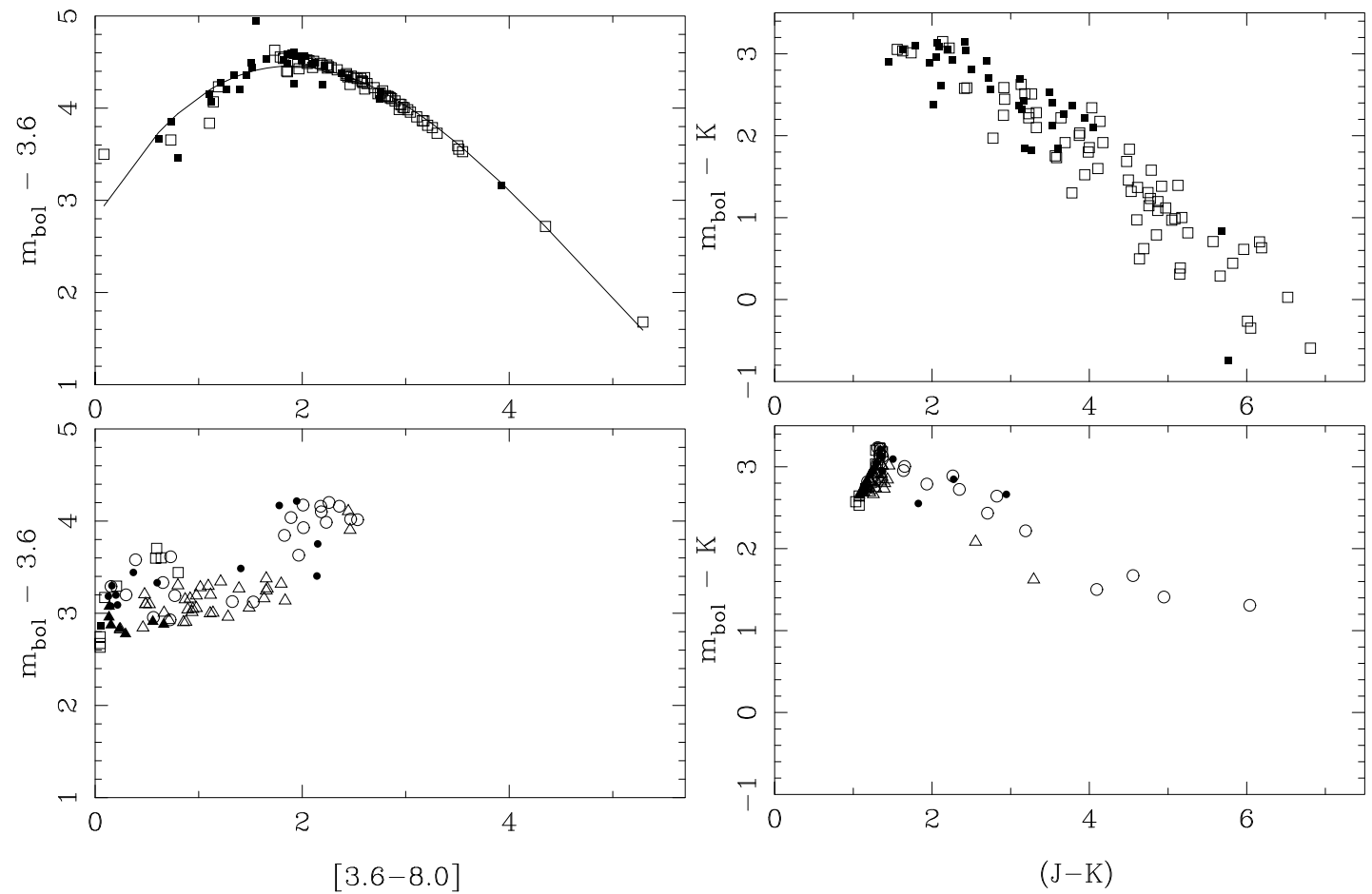

Fig. 12. The bolometric correction at $3.6 \mu \mathrm{m}$ versus [3.6]-[8.0] colour (left) and $K$ versus $J-K$ colour (right) for C stars (top panel) and M stars (bottom panel). SMC objects are plotted as filled squares in the top panel. In the bottom panel, foreground objects are plotted as squares, supergiants as triangles and AGB stars as circles. SMC objects are plotted with filled symbols. The curved line in the top right panel is the fit to the data: $m_{\text {bol }}=2.78+1.926([3.6-8.0])-0.6309([3.6-8.0])^{2}+0.04239([3.6-8.0])^{3}$.

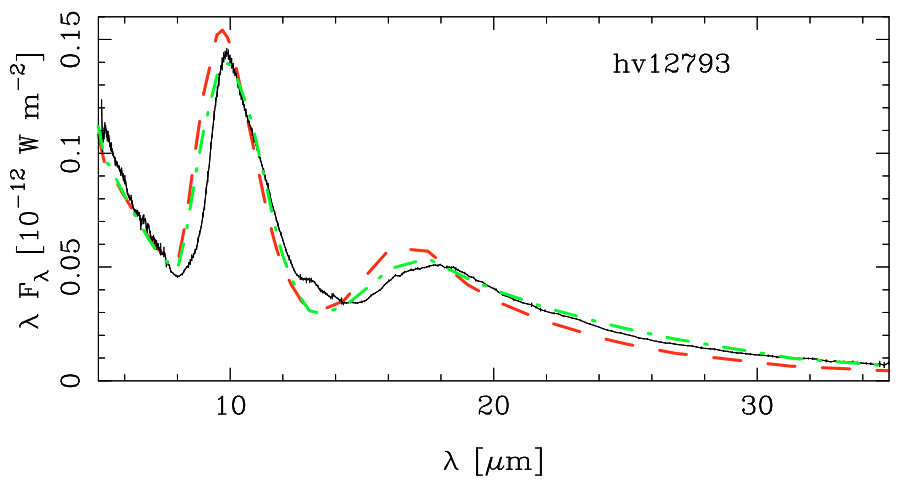

Fig. 13. The effect of grain shape, showing the original best-fitting model with small spherical olivine grains (red dashed line) and with a CDS (green dot-dashed line), both with $5 \%$ iron. Models assuming a CDE or DHS give results nearly identical to the CDS model.

do change: from $L=118000 L_{\odot}$ and $\dot{M}=5.1 \times 10^{-7} M_{\odot} \mathrm{yr}^{-1}$ for $f_{\max }=0.4$ to $L=136000 L_{\odot}$ and $\dot{M}=3.3 \times 10^{-7} M_{\odot} \mathrm{yr}^{-1}$ for $f_{\max }=0.9$.

We conclude that, compared to the small-particle limit, a distribution of shapes shifts the peak of the 9.8 and $18 \mu \mathrm{m}$ feature to slightly longer wavelengths, in better agreement with observations. Assuming porous grains achieves the same effect. When considering grains of finite size and a DHS we find for HV 12793 that a grain size of around $1 \mu \mathrm{m}$ fits the observations. This result is typical of many of the M giants in the sample, although the porosity is not well constrained.

Höfner (2008) recently studied the winds of M giants using a dynamical atmosphere and wind models and concluded that radiation pressure is sufficient to drive the outflow if the restriction of small particles is relaxed. She also showed that grain growth

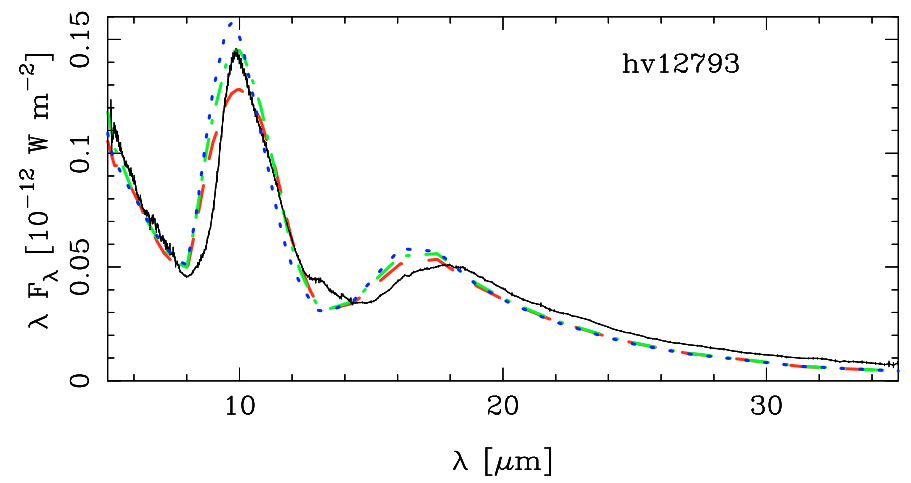

Fig. 14. The effect of grain size for homogeneous spheres (95\% olivine and $5 \%$ iron). Grain sizes are 1.5 (red dashed line), 1.2 (green dotdashed line) and $0.6 \mu \mathrm{m}$ (blue dotted line). Grains smaller than $0.6 \mu \mathrm{m}$ are indistinguishable from the $0.6-\mu \mathrm{m}$ case.

naturally halts at particle sizes of about $1 \mu \mathrm{m}$. The present study provides observational support to these theoretical conclusions.

\subsection{Dust in the C-stars}

\subsubsection{Silicon carbide}

While amorphous carbon dominates the dust produced by carbon stars (e.g. Martin \& Rogers 1987), silicon carbide (SiC) produces the most recognisable dust emission feature at $\sim 11.3 \mu \mathrm{m}$ (Treffers \& Cohen 1974, and references therein). The models here utilise the SiC optical constants of Borghesi et al. (1985) and Pégourié (1988). In reality, more complex situations may occur than assumed in the standard model, namely the coexistence of two grains ( $\mathrm{SiC}$ and $\mathrm{AMC}$ ) at the same temperature (adding 


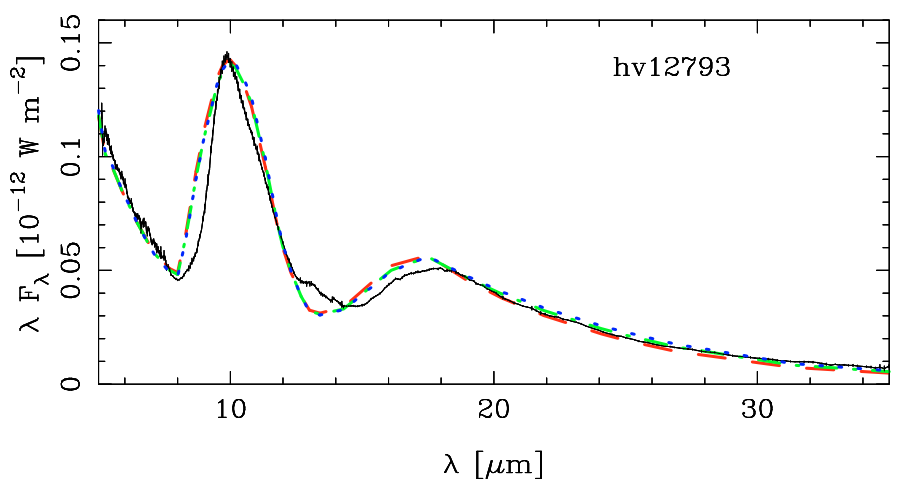

Fig. 15. The effect of porosity using a DHS with $f_{\max }=0.4$ (red dashed line), 0.7 (green dot-dashed line) and 0.9 (blue dotted line) for a grain size of $1.2 \mu \mathrm{m}$.

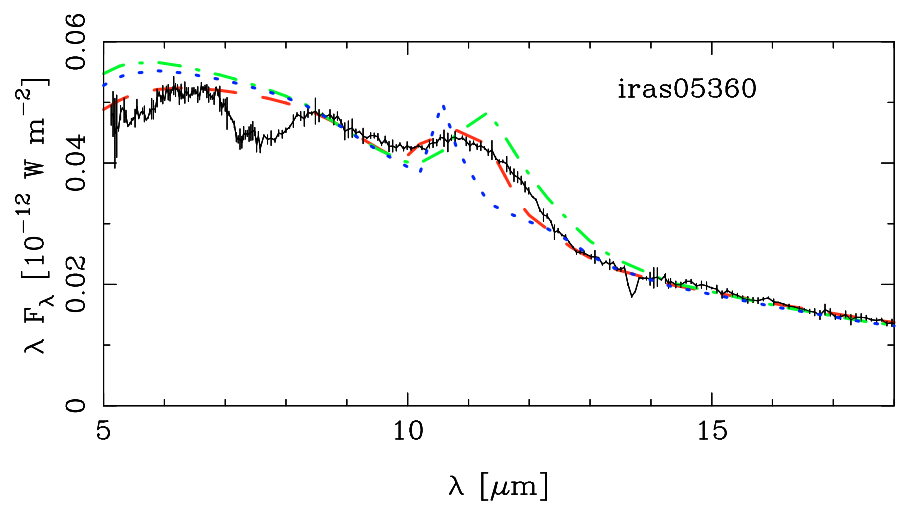

Fig. 16. A comparison of different types of $\mathrm{SiC}$ assuming small spherical particles: $8 \% \beta$-SiC from Borghesi et al. (1985) (red dashed line), $8 \% \alpha$-SiC from Pégourié (1988) (green dot-dashed line), and $2 \% \beta$-SiC from Pitman et al. (2008) (blue dotted line), with the remaining fraction of the dust being AMC. The models are tied to the observed spectrum based on the flux density in the $8.5-9.5 \mu \mathrm{m}$ region.

the absorption coefficients in proportion) in the small particle limit.

Some of the possible effects are illustrated here, using IRAS 05360-6648 as the test case. Figure 16 compares the best fit using $8 \% \beta$-SiC from Borghesi et al. (1985), $8 \% \alpha$-SiC from Pégourié (1988), and $2 \% \beta$-SiC from Pitman et al. (2008), assuming small spherical particles. The constants from Borghesi et al. fit the data best; the other two are too sharply peaked compared to the observations.

Figure 17 shows the effects of the CDE and CDS approximations. Since the SiC from Pégourié (1988) peaks to the right of that of Pitman et al. (2008) this effect is enhanced when using a distribution of shapes, and the latter clearly provides the better fit. On the other hand, the shape of the feature becomes "boxy" which is not observed.

Figure 18 shows the effect of using DHS with different values of $f_{\max }$ in the case of $\beta$-SiC from Pitman et al. (2008). The model with $f_{\max }=0.8$ fits the data reasonably well and slightly better than the CDE and CDS approximation, but still there is a lack of emission on the blue side of the feature.

Kozasa et al. (1996), and more recently Papoular (2008), considered grains with a core of $\mathrm{SiC}$ and a mantle of AMC, as an explanation for the observed variations in the strength of the $\mathrm{SiC}$ feature. Figure 19 shows the best-fitting models for grains with a $0.01 \mu \mathrm{m} \mathrm{SiC} \mathrm{core,} \mathrm{and} \mathrm{a} \mathrm{total} \mathrm{radius} \mathrm{of} 0.03,0.04,0.08 \mu \mathrm{m}$. The figure shows that changing the volume fraction of the $\mathrm{SiC}$

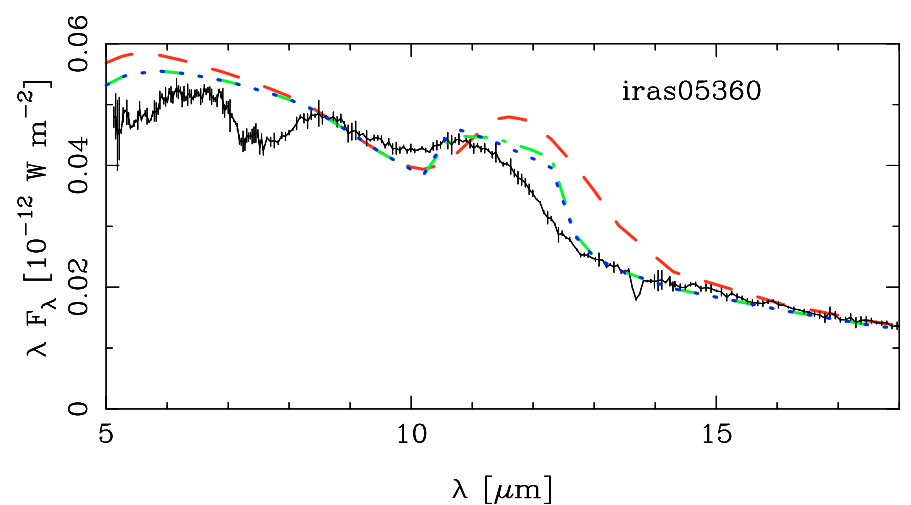

Fig. 17. The effect of shape distribution: $\mathrm{CDE}$ with $6 \% \beta$-SiC from Pégourié (1988) (red dashed line), CDE (green dot-dashed line), and CDS (blue dotted line) with $2 \% \beta$-SiC from Pitman et al. (2008), with the remaining fraction being AMC.

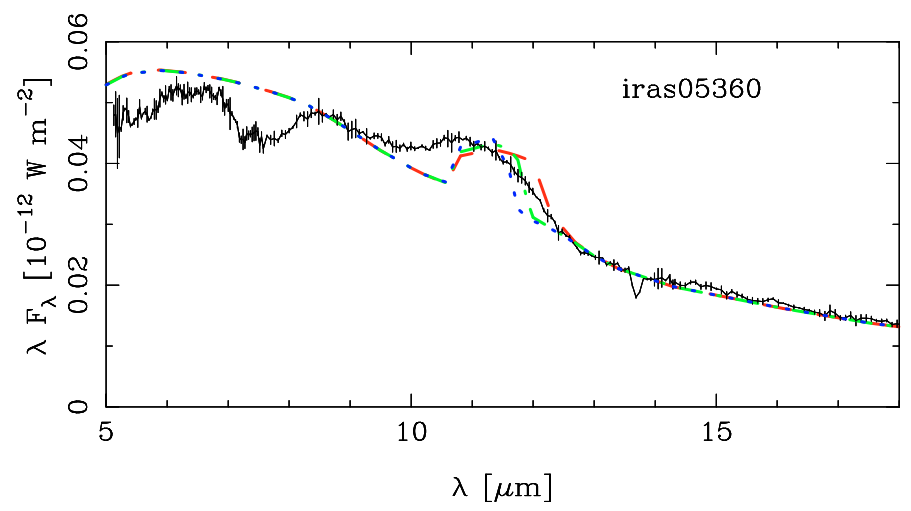

Fig. 18. The effect of using a DHS with $f_{\max }$ equal to 0.9 (red dashed line), 0.8 (green dot-dashed line), and 0.7 (blue dotted line), with $1 \%$ $\beta$-SiC from Pitman et al. (2008) and 99\% AMC.

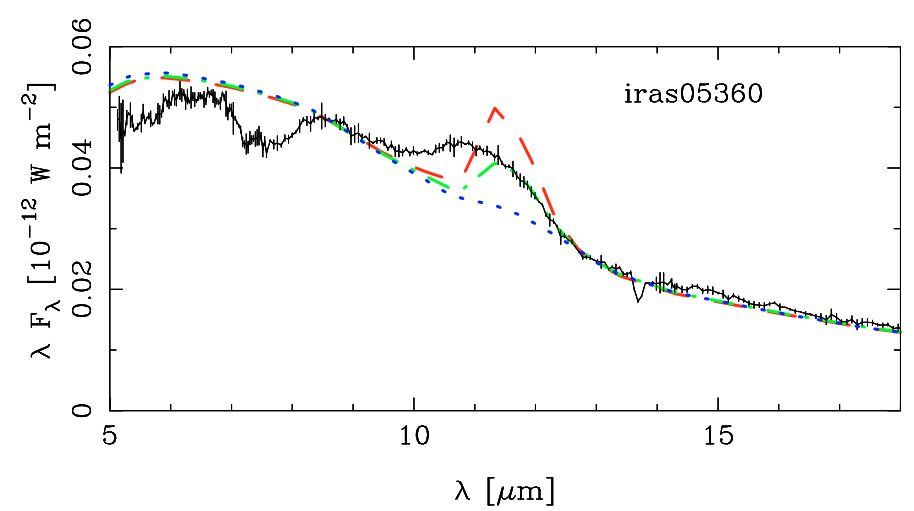

Fig. 19. The effect of using spherical core-mantle grains, with a $\mathrm{SiC}$ core of $0.01 \mu \mathrm{m}$, and a total radius of 0.03 (red dashed line), 0.04 (green dot-dashed line), and 0.08 (blue dotted line). The volume fraction of $\mathrm{SiC}$ in these grains is $\sim 4,2$, and $0.2 \%$, respectively.

core from about 4 to 2 to $0.2 \%$ changes the strength of the $\mathrm{SiC}$ feature from stronger than in any spectrum in the present sample to effectively zero. In the test calculation by Papoular, the grain reached its final size about one year after its initial condensation, with a core volume of $12 \%$, which would produce a $\mathrm{SiC}$ feature stronger than any in the sample considered here. Assuming an expansion velocity of $10 \mathrm{~km} \mathrm{~s}^{-1}$, a dust particle would travel about 1 stellar radius per year in the case of IRAS 05360-6648. The formation of the coating must therefore proceed much more 
efficiently than in the test calculation of Papoular for core-mantle grains to be a viable option for explaining the maximum observed strength of the SiC feature. Speck et al. (2009) note only one case where presolar $\mathrm{SiC}$ grains are found in the cores of carbon grains in meteoritic samples, adding further doubt about the importance of grains with $\mathrm{SiC}$ cores and $\mathrm{AMC}$ mantles.

Lagadec et al. (2007) argue for a $\mathrm{SiC} \rightarrow \mathrm{C}$ condensation sequence in Galactic C-stars and Leisenring et al. (2008, their Fig. 13) discuss three condensation sequences, two of which start with $\mathrm{SiC}$ cores. The absence of a very strong $\mathrm{SiC}$ feature in any Galactic and MC C-star predicted for a core-mantle grains with even moderate $\mathrm{SiC}$ volume fraction appears to rule out condensation sequences II and III in Leisenring et al. (2008). Leisenring et al. also argue that $\mathrm{SiC}$ and $\mathrm{C}$ condense nearsimultaneously. In that case a mix of condensation sequences I ( $\mathrm{SiC}$ on top of $\mathrm{C}$ ) and II could take place.

\subsubsection{Magnesium sulfide}

In their sample Leisenring et al. (2008) found 6 out of 29 (21\%) C-stars in the SMC to show MgS, and 27/44 =61\% stars in the LMC (and 14/34 = 41\% for Galactic sources). In our larger sample the ratio for the SMC now becomes $8 / 33=24 \%$, and $48 / 68=71 \%$ for the LMC.

Only one of the 22 stars with $R_{\text {outer }}<50 R_{\text {inner }}$ show MgS. (The exception is MSX LMC 1213 with $R_{\text {outer }}=41 R_{\text {inner. }}$ ) The dust temperature at the outer radius ranges from 175 to $400 \mathrm{~K}$ in these stars, suggesting that $\mathrm{MgS}$ forms at temperatures below $400 \mathrm{~K}$, consistent with the $\mathrm{MgS}$ temperatures derived by Hony et al. (2002).

Recently, Zhukovska \& Gail (2008) studied the condensation of $\mathrm{MgS}$ in the outflows of C-stars using thermo-chemical models including grain growth. They preferred a scenario where $\mathrm{MgS}$ mantles grew on $\mathrm{SiC}$ cores. Unfortunately, they did not consider the case of $\mathrm{SiC}+\mathrm{AMC}+\mathrm{MgS}$. However, as $\mathrm{MgS}$ forms at the lowest temperatures, one could expect to have grains consisting of a SiC core, an inner mantle of AMC (in spite of the remarks in the previous section) and an outer mantle of $\mathrm{MgS}$. Alternatively, a model with pure AMC grains on the one hand and grains with a $\mathrm{MgS}$ mantle on a core of $\mathrm{SiC}$ on the other hand are considered below. Some test calculations are done for such grains ${ }^{7}$. Our model does not permit a gradient in grain properties within the circumstellar dust shell; thus we cannot account for the possibility that grains might have an outer layer of $\mathrm{MgS}$ only in the outerparts of the shell. We remind the reader that the presence of $\mathrm{MgS}$ may mask the $\mathrm{SiC}$ strength, making our estimates of the $\mathrm{SiC}$ mass fraction a lower limit in spectra showing $\mathrm{MgS}$ emission.

Hony et al. (2002) showed the importance of grain shape in fitting $\mathrm{MgS}$ to the observed $30 \mu \mathrm{m}$ feature. They found that a CDE provided a good fit. Here we also consider CDE, and in this respect one would not favour core-mantle grains with $\mathrm{MgS}+\mathrm{SiC}+\mathrm{AMC}$ (see Fig. 20). The Mie-code employed also provides the optical constant of the aggregate following EMT, and we used this to calculate the absorption coefficients in a CDE. As MgS is a minor species, the effect of broadening the $\mathrm{MgS}$ feature as seen in the models of Hony et al. does not occur. In this respect, AMC as one grain, with a separate coremantle grain of $\mathrm{SiC}+\mathrm{MgS}$ fits the data better. The volume fraction of $\mathrm{MgS}$ in such a grain is substantial, and using the optical

\footnotetext{
7 Adapting the code for $n$-layered spherical grains available at http://wwW . astro. spbu.ru/JPDOC/CODES/NMIE/n-miev3a. for
}

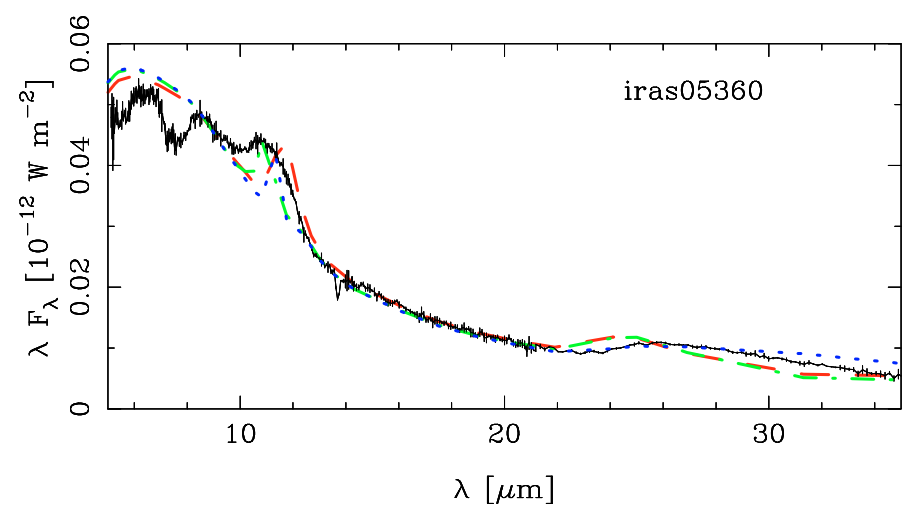

Fig. 20. Some models that include MgS. (1) Core with $2 \% \mathrm{SiC}$, inner mantle with $91 \%$ AMC, outer mantle with $7 \% \mathrm{MgS}$. CDE using the optical constants calculated using EMT (red dashed line), (2) two grain model, $90 \% \mathrm{AMC}$ and $10 \%$ of a core-mantle grain with $45 \% \mathrm{SiC}$ core and 55\% MgS mantle (green dot-dashed line), and (3) two grain model, $94 \%$ AMC and $6 \%$ of a core-mantle grain with $3 \% \mathrm{SiC}$ core and $97 \% \mathrm{MgS}$ mantle, assuming CDE using the optical constants calculated using EMT (blue dotted line).

constants of the aggregate in the $\mathrm{CDE}$ approximation does broaden the feature sufficiently.

\subsection{Mass-loss and stellar evolution}

This section compares the derived MLRs and other quantities by comparing the observations with the AGB models of Vassiliadis \& Wood (1993, hereafter VW) and synthetic models based on recipes developed by Wagenhuber \& Groenewegen (1998, hereafter WG), tuned to reproduce the VW models, as explained in Appendix C of Groenewegen \& Blommaert (2005). This last option allows us to investigate initial masses and MLRs different from those available from the VW models alone (with a maximum initial mass of $5 M_{\odot}$ ). From VW we take the models with $Z=0.008$ as representative of the LMC, since they show qualitatively similar behaviour. Using the WG implementation of the VW models, we have calculated a model for $7.9 M_{\odot}$, which is the largest mass for which the WG models converge. In addition we have used the WG recipes to calculate models using the Reimers law with a scaling factor of 5 (hereafter the Reimers models), which Groenewegen \& de Jong (1993) advocated to fit many of the observables of $\mathrm{C}$ stars in the LMC. The major divergence is the recipe to determine the MLR on the AGB. In the case of the Reimers law this is $\dot{M} \sim L R / M$, while in the case of VW it is basically the minimum of the single scattering limit $\dot{M}=2.02 \times 10^{-8} L / v_{\text {exp }}$, and an empirical relation between $\log \dot{M}$ and $P$. The (fundamental mode) period, $P$, is calculated from a period-mass-radius relation (see VW for details).

Figure 21 shows the relation between MLR and luminosity, with the VW and Reimers model tracks overplotted (the crosses connected with the dotted lines). From the individual evolutionary tracks, a model is plotted every 5000 years. The density of points is therefore representative for the time spent at the certain position in the diagram. It also explains the "excursions" which are due to the finite probability of catching a star during a thermal pulse or during the "luminosity dip" that follows. Models are plotted for initial masses of 1.5, 2.5, 5.0 and 7.9 $M_{\odot}$, which evolve at increasing luminosity. The Reimers $1.5 M_{\odot}$ model is only identified by the few points near $\log L=3.5 L_{\odot}$ and MLRs below $10^{-7} M_{\odot} \mathrm{yr}^{-1}$. 

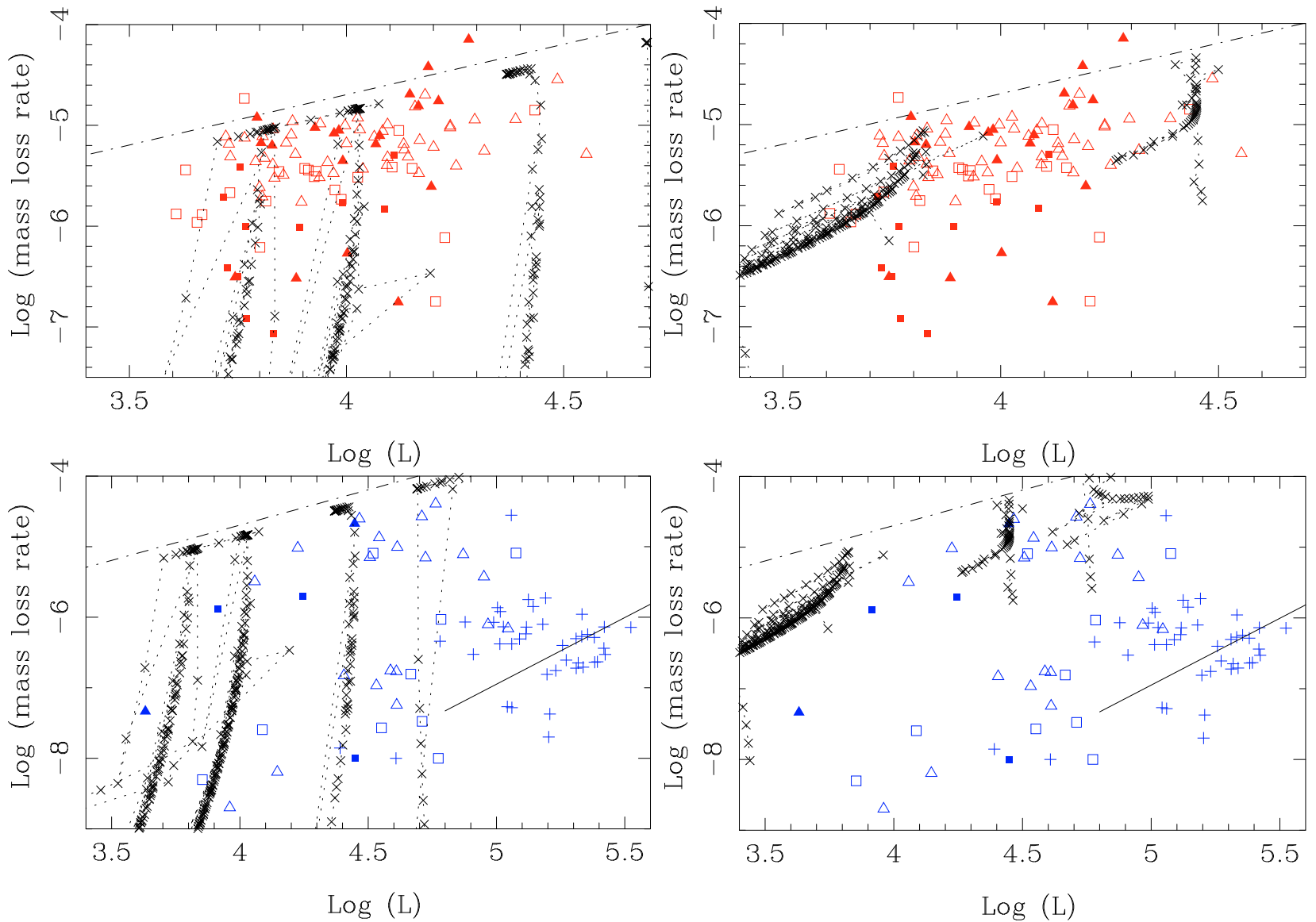

Fig. 21. Mass-loss rate versus luminosity for C stars (top panel, red colours), M stars (bottom panel, blue colours), VW models (left), and Reimers models (right). AGB stars in the SMC are plotted as squares, in the LMC as triangles. Objects with Mira like pulsation amplitudes are plotted with open symbols, objects with smaller amplitudes as filled symbols. RSG are plotted as plus-signs independent of host galaxy and pulsation amplitude. The VW and Reimers models are plotted as crosses connected by the dotted line for initial masses of 1.5, 2.5, 5.0 and 7.9 $M_{\odot}$, but not every track is visible in every panel. Each cross represents a time interval of 5000 years. The dot-dashed line indicates the single scattering limit for a velocity of $10 \mathrm{~km} \mathrm{~s}^{-1}$. The solid line is the relation found by Verhoelst et al. (2009) for Galactic RSG.

Several conclusions can be drawn. From the observational point of view, the MLRs of the C stars in the sample are located in a strip with a width of about 1 dex, slightly lower than the single-scattering limit. At the lowest MLRs, the majority of stars have smaller amplitudes (filled symbols). The dot-dashed line indicates that the single-scattering limit for a velocity of $10 \mathrm{~km} \mathrm{~s}^{-1}$ which is the same velocity as adopted in the RT models. However there is an additional systematic uncertainty in the $y$-scale because of the adopted dust-to-gas ratio in the RT modelling.

The MLR distribution for the O-rich AGB stars is less clear. One could describe two sequences, one of low MLRs $\left(10^{-7}-10^{-6} M_{\odot} \mathrm{yr}^{-1}\right)$ for $\log L \approx 3.7-4.7$ and one of large MLRs $\left(3 \times 10^{-6}-3 \times 10^{-5} M_{\odot} \mathrm{yr}^{-1}\right.$ for $\left.\log L \approx 4.1-4.8\right)$, which is roughly consistent with the VW models, as discussed below.

The MLRs of the RSG scatter around the relation of Galactic RSG recently derived by Verhoelst et al. (2009).

The comparison to the evolutionary models is interesting, and favours overall the mass-loss recipe adopted in VW. Taking $\mathrm{M}$ and $\mathrm{C}$ stars together it is clear from Fig. 21 that there is a large scatter in MLR for a given luminosity. This is clearly not predicted by the Reimers models, where the luminosity fixes the MLR. The VW models do predict a large variation of MLR at a given luminosity, as observed, and consistent with other evolutionary considerations. The majority of $\mathrm{C}$ stars are bound between the 1.5- and 5.0- $M_{\odot}$ models, which indeed is the range where one believes that C-stars form in the LMC (Groenewegen \& de Jong 1993). The lowest luminosity C-stars in the sample are located in the SMC (the open squares) where the initial mass to become a C star is slightly lower then in the LMC (Groenewegen 1993). The VW models also explain that, in the $1.5-5-M_{\odot}$ mass range, the $\mathrm{M}$ stars have lower MLRs than the $\mathrm{C}$ stars at earlier times in their evolution.

There are essentially no C stars brighter than $\log L=4.5$, but many $\mathrm{M}$ stars are brighter than that, and they span a large range in MLRs. The separation near $5 M_{\odot}$ is thought to be due to HBB (Smith et al. 1995). The 7.9 $M_{\odot}$ model based on the VW mass-loss recipe nicely passes through the data.

Although the VW models generally provide a qualitatively correct picture of mass-loss evolution along the AGB for both M and $\mathrm{C}$ stars, they fail on one point. If the sample under study were complete, then the distribution of observed data should match the distribution of the crosses, but this is clearly not the case. While the observations give a fairly uniform distribution of MLRs at a given luminosity for the C-stars, the VW models predict that most of the MLRs are in fact limited by the singlescattering limit. As in VW the expansion velocity is assumed to be a function of period. With a maximum of $15 \mathrm{~km} \mathrm{~s}^{-1}$, the maximum MLR in the VW is slightly lower than the dot-dashed line which represents the single-scattering limit for $10 \mathrm{~km} \mathrm{~s}^{-1}$.

One could argue that this a a selection effect and that the present sample is incomplete for the low MLRs and lowest 

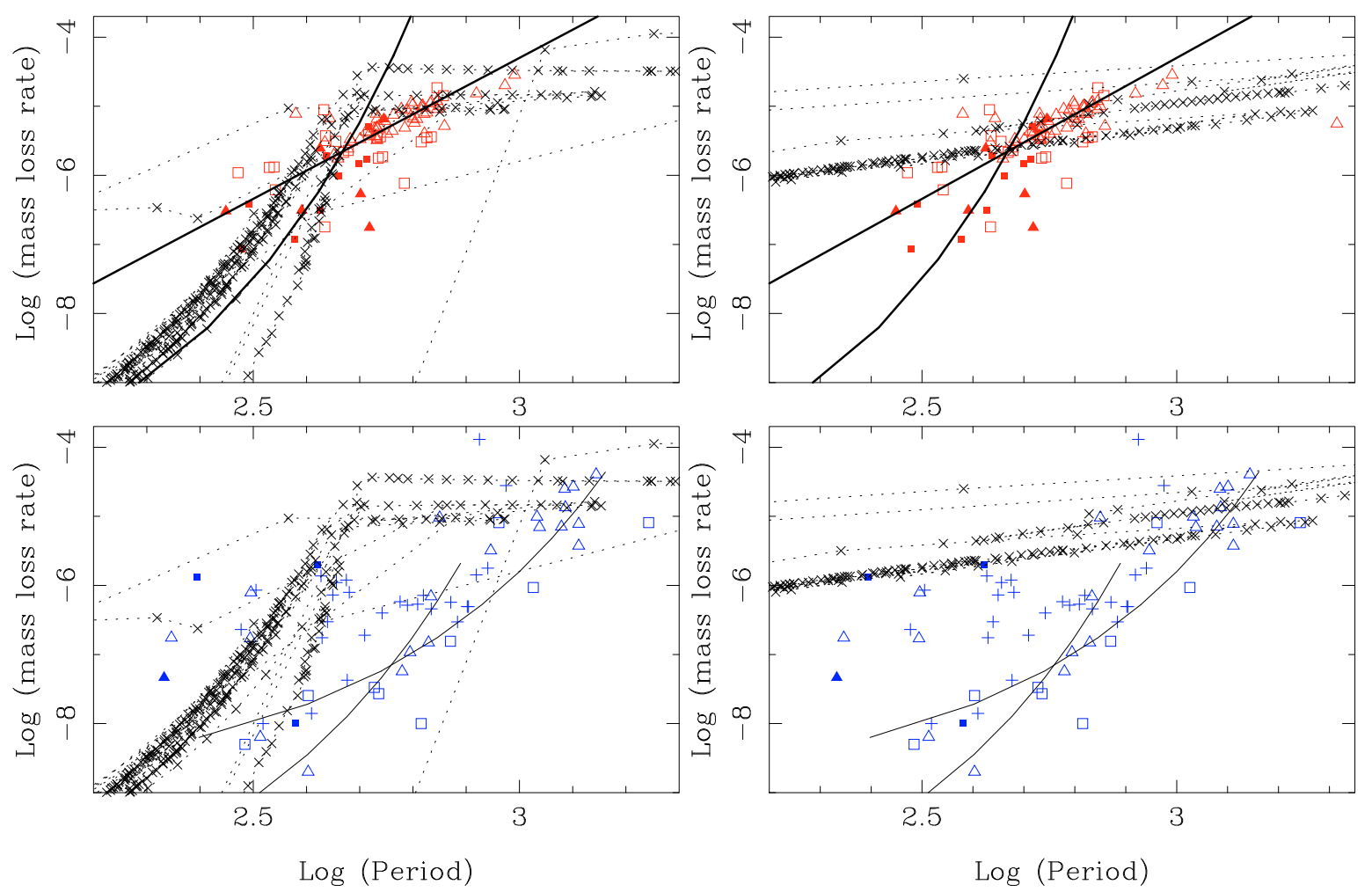

Fig. 22. Mass-loss rate versus period for C stars (top panel, red colours), M stars (bottom panel, blue colours), VW models (left), and Reimers models (right). Symbols are as in Fig. 21. In the upper panels, the curved solid line indicates the MLR-period relation from VW, the straight solid line the relation derived for Galactic C-rich Miras by Groenewegen et al. (1998). In the bottom panel, the two lines indicate a fit to most of the M stars, and the VW relation for a period 0.6 times the pulsation period.

luminosities, which would predominantly be O-rich stars. It was verified however, by comparing the cumulative absolute magnitude distribution function of the C-stars in this sample to the sample of about $1800 \mathrm{C}$-stars selected on colour from the entire SAGE survey by Matsuura et al. (2009), and using the relation in Fig. 12 to transform colours to bolometric magnitudes, that the present C-star sample is unbiased in absolute magnitude down to $M_{\text {bol }} \approx-5.5(\log L=4.0)$.

Figure 22 shows the derived MLR plotted against pulsation period, for the C stars and M stars, with the VW and Reimers models overplotted. Overall, the VW models cover the area occupied by the $\mathrm{C}$ stars, except in the early phases of evolution when the stars are not yet carbon-rich. The Reimers models do not cover the range in mass-loss of the $\mathrm{C}$ stars. It is surprising that the $\mathrm{M}$ stars do not follow the VW relation, even though it was derived for a sample that included O-rich Mira variables. A relation similar to Eq. (2) of VW that fits most of the O-rich data is $\log \dot{M}=-9.0+0.0032 P$. An alternative interpretation is that the largest MLRs of the M stars are consistent with the VW model, namely that they are on the horizontal part of the most massive evolutionary tracks, and that the lower MLRs are roughly consistent with a track parallel to the VW relation for a period roughly 0.6 times the pulsation period. A connection with overtone pulsation comes to mind although the amplitudes of these $\mathrm{M}$ stars indicate Mira-like (fundamental-mode) pulsations.

Figure 23 plots the MLR versus the observed or estimated pulsation amplitude in the $I$-band. Whitelock et al. (2003) showed a similar diagram with MLR vs. the $K$-band. For the larger amplitudes there is a reasonably well defined and almost flat relation. Outside the regime of Mira-like pulsation there is
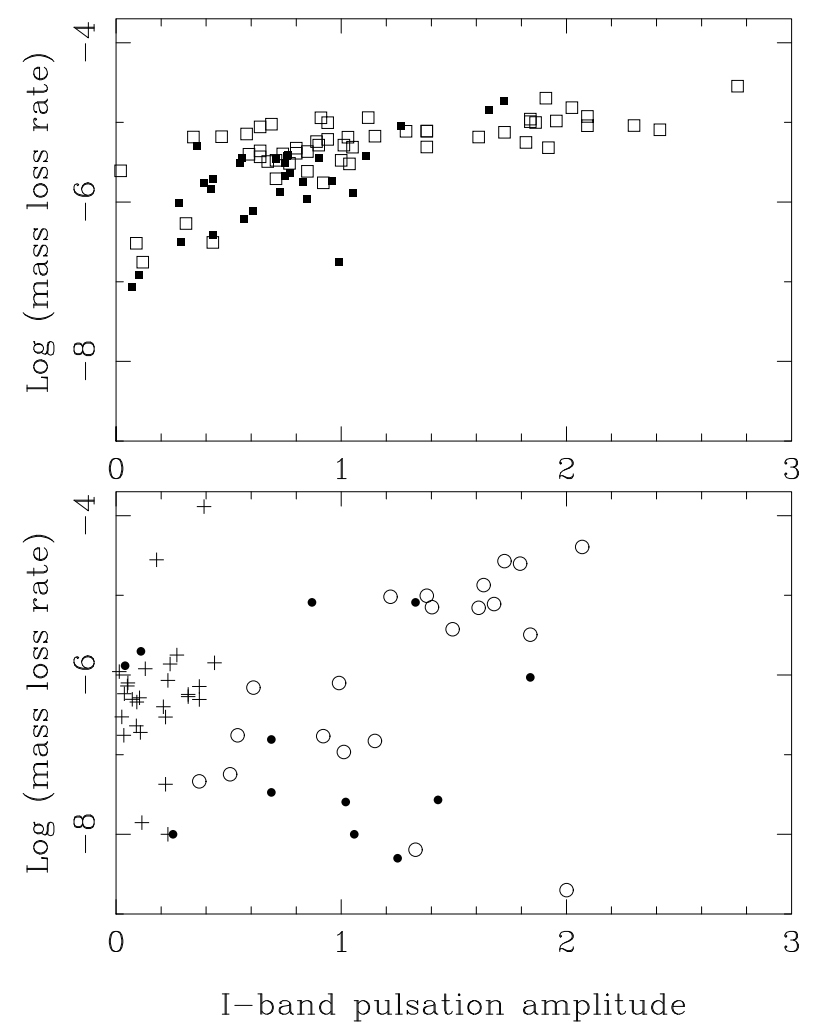

Fig. 23. Mass-loss rate versus (estimated or measured) $I$-band pulsation amplitude for $\mathrm{C}$ stars (top panel) and $\mathrm{M}$ stars (bottom panel). C stars in the LMC are plotted as open squares, in the SMC as filled squares. O-rich AGB stars in the LMC are plotted as open circles, and in the SMC as filled circles. RSG are plotted as plus signs. 

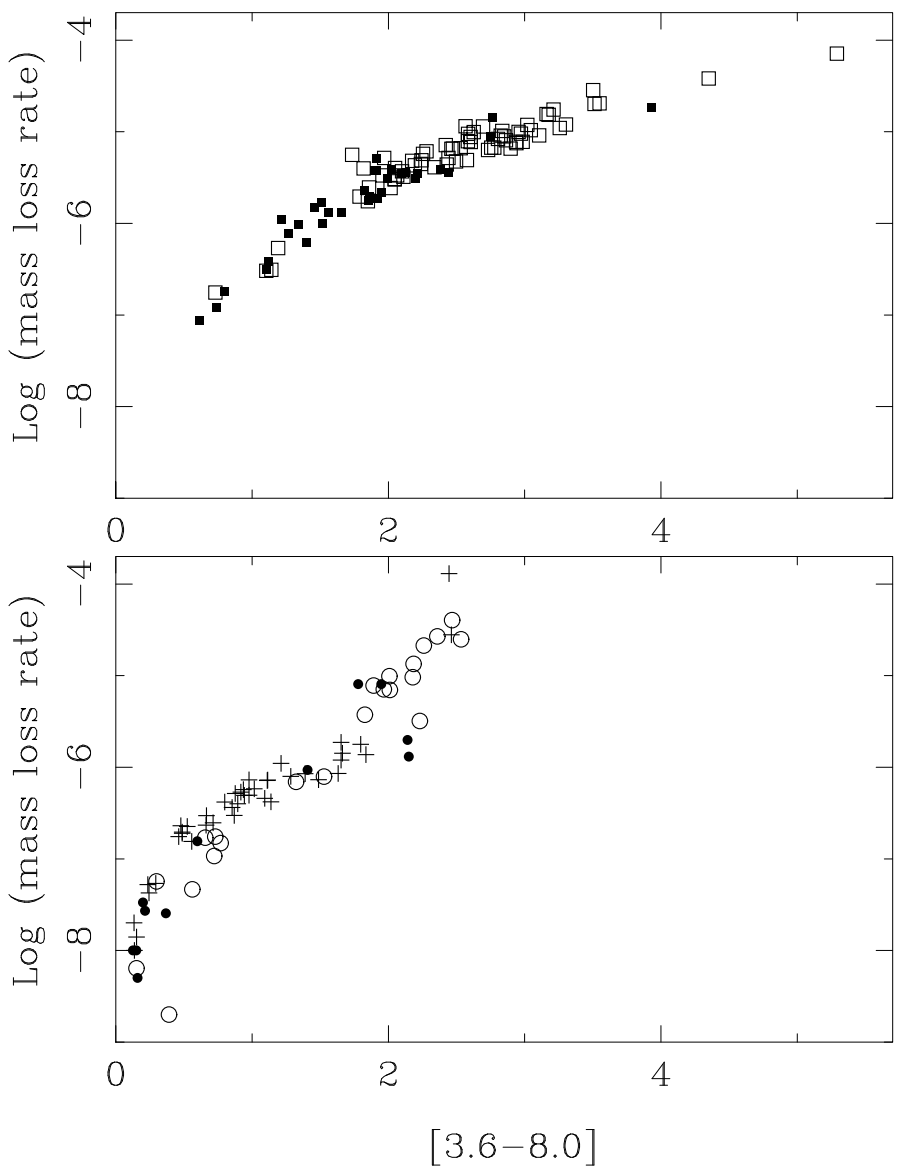

Fig. 24. Mass-loss rate versus colour for C-stars (top panel), and Mstars (bottom panel). Symbols as in Fig. 23.

much more scatter. For the $\mathrm{M}$ stars there may be a trend of larger MLRs with larger amplitudes that is steeper than for the C stars but there is even more scatter.

Figure 24 plots the MLR as a function of [3.6]-[8.0] colour. Whitelock et al. (2003) presented a similar diagram with MLR vs. $[\mathrm{K}]-[12]$. Generally, redder colours are associated with larger MLR, as expected. For C stars the relation is tight, with no dependence on metallicity apparent (assuming that the expansion velocity and dust-to-gas ratio are on average the same in the LMC and SMC).

For the M stars there appear to be two relations, with both RSGs and AGB stars following the same relation up to a certain colour, with a discontinuity for stars that are associated with the final stages of evolution of the most massive intermediate stars according to the VW models (see Fig. 21). Together with the relations in Fig. 12, the [3.6]-[8.0] colour can be used to estimate luminosity and MLR.

Sloan et al. (2008) presented two tools to measure the amount of dust in their sample of evolved oxygen-rich Magellanic stars. The first, dust emission contrast (DEC) was originally introduced by Sloan \& Price (1995), and is the ratio of the dust emission to stellar emission from 7.67 to $14.03 \mu \mathrm{m}$, based on a stellar photosphere fitted to the spectrum over the range $6.8-7.4 \mu \mathrm{m}^{8}$. The second was the [7]-[15] color, integrated at 6.8-7.4 and $14.4-15.0 \mu \mathrm{m}$, wavelength ranges chosen to avoid molecular absorption from $\mathrm{H}_{2} \mathrm{O}$ at $6.6 \mu \mathrm{m}$ and $\mathrm{SiO}$, starting at $7.5 \mu \mathrm{m}$, and dust emission features from silicates and

\footnotetext{
${ }^{8}$ Sloan et al. (2008) shifted this range for the IRS data from the range originally defined for the LRS data.
}
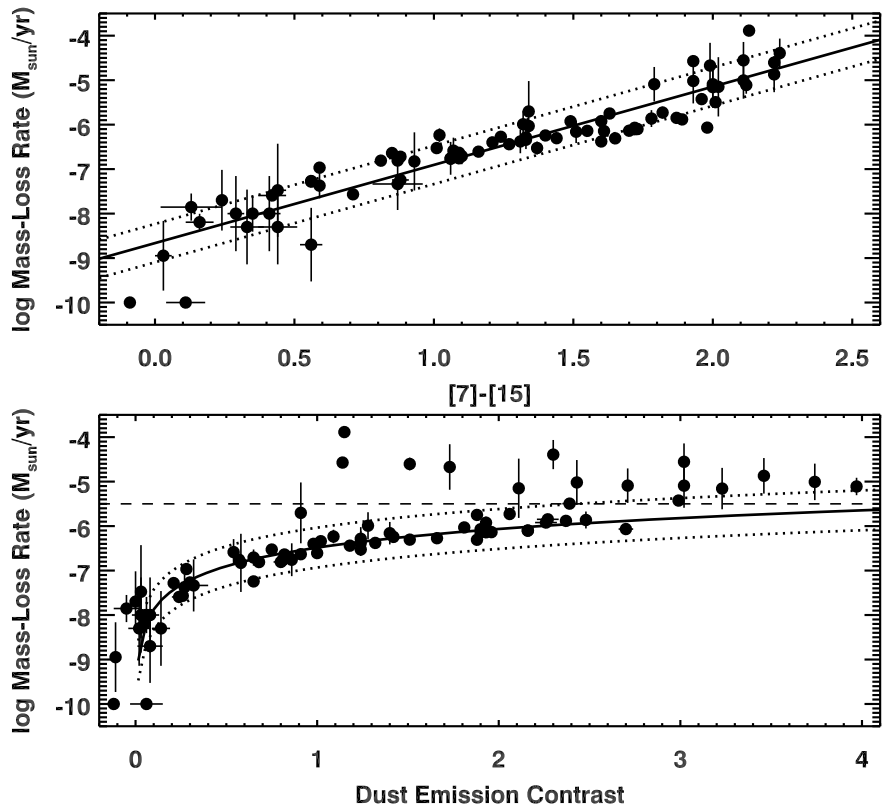

Fig. 25. Mass-loss rate as a function of [7]-[15] color (top panel) and DEC (dust emission contrast; bottom panel). In both panels, the solid line plots the functions given in Eqs. (5) and (6), and the dotted lines show the average uncertainty, which is formally the standard deviation of the difference between the actual data and the fitted function. The dashed line in the bottom panel separates the data excluded from the fit. Also excluded were the four points with negative DECs. Neither panel includes foreground objects.

alumina. Figure 25 plots the mass-loss rates derived here with these quantities obtained from the IRS data. The mass-loss rates follow the [7]-[15] color closely:

$\log \dot{M}=1.759$ ([7] - [15]) - 8.664,

with an average uncertainty of $0.43 \mathrm{dex}$, or $27 \%$ of the massloss rate. Thus, the [7]-[15] color provides an excellent means of estimating mass-loss rates from infrared spectra, provided the dust is configured in an outflowing shell as opposed to a disc.

Figure 25 also plots the mass-loss rate as a function of DEC, and the relation is more complex than with [7]-[15] color. The DEC was originally defined for optically thin dust shells, and it breaks down as a useful measure when the dust grows optically thick. Once mass-loss rates exceed $\sim 10^{-5.5} M_{\odot} \mathrm{yr}^{-1}$, the $10 \mu \mathrm{m}$ silicate emission feature will begin to self-absorb, and the DEC will begin to decrease, even as the mass-loss rate grows. The [7]-[15] color is thus a more robust measure. There is still a relation between mass-loss rate and DEC, provided certain caveats are kept in mind. Fitting the data where the DEC is positive and the mass-loss rate is less than $10^{-5.5} M_{\odot} \mathrm{yr}^{-1}$ gives the relation

$\dot{M}=3.281 \times 10^{-7}(\mathrm{DEC})^{1.392}$

with an average uncertainty of $0.45 \mathrm{dex}$, or $28 \%$ of the mass-loss rate. As Fig. 25 shows, though, the data do not fit this relation well when the DEC exceeds $\sim 2.5$.

One must keep in mind that these mass-loss rates assume a gas-to-dust ratio of 200, since that is the value assumed in the radiative transfer modelling. The spectroscopy really measures the dust, so it would be appropriate to scale these relations accordingly for different gas-to-dust ratios.

Figure 26 shows the bolometric $P L$-relation with the VW and Reimers models overplotted, together with various (extrapolated) PL-relations for C- and O-stars (Feast et al. 1989; Hughes \& Wood 1990; Groenewegen \& Whitelock 1996). 

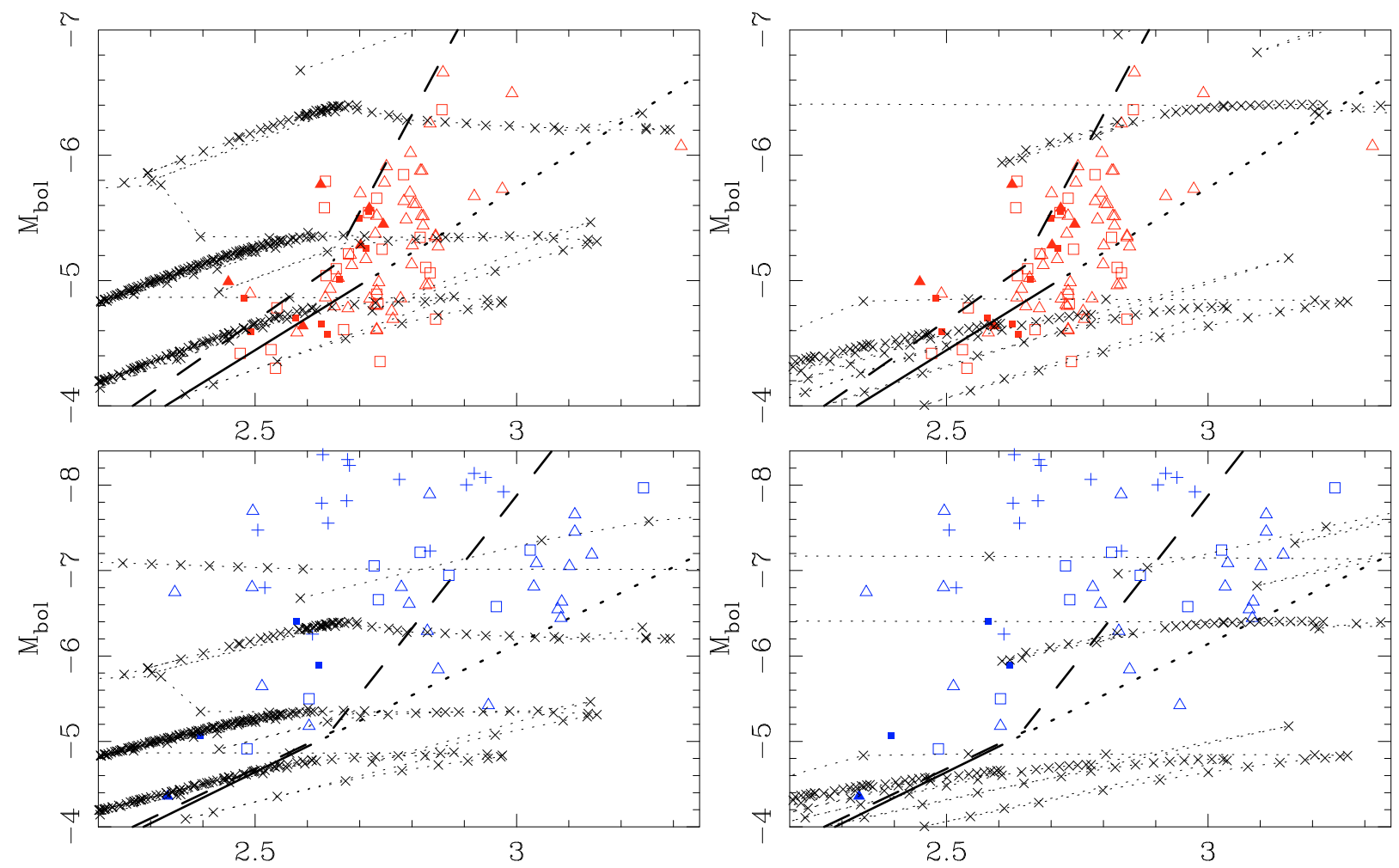

$\log$ Period

$\log$ Period

Fig. 26. Bolometric magnitude versus pulsation period for C-stars (top panel, red colours), and M-stars (bottom panel, blue colours), and for VW models (left) and Reimers models (right). AGB stars in the SMC are plotted as squares, in the LMC as triangles. Objects with Mira like pulsation amplitudes are plotted with open symbols, and filled symbols otherwise. RSG are plotted as plus-signs independent of host galaxy and pulsation amplitude. For the C-star panels, the solid line indicates the PL-relation from Groenewegen \& Whitelock (1996) and the dotted line the extrapolation beyond $\log P=2.7$. For the O-star panels, the solid line indicates the $P L$-relation from Feast et al. (1989) and the dotted line the extrapolation beyond $P=400$ days. The dashed line with the break and discontinuity at $\log P=2.65$ in both panels is the $P L$-relation from Hughes \& Wood (1990) based on a sample dominated by O-stars.

Most of the VW points are to the left of the data points, which can be interpreted as meaning that pulsation in the fundamental period is only reached towards the end of AGB evolution, which is consistent with the findings of Lebzelter \& Wood $(2005,2007)$ for the clusters 47 Tuc and NGC 1846.

Figure 27 shows the data in a different light, only plotting the large amplitude (Mira) variables, and highlighting the knowledge regarding the presence of Lithium, an indicator of HBB (data from Smith \& Lambert 1989, 1990; Smith et al. 1995). It shows the well known result that the known Li-rich stars are Orich stars located in the interval $-7.2 \lesssim M_{\text {bol }} \lesssim-6.0$ (see the discussion and Fig. 6 in Smith et al. 1995). The C-star marked as undergoing HBB is IRAS 04496-6958 which was suggested to be in that state by Trams et al. (1999). At that time ISO spectra suggested a silicate dust shell, although the central star is C-rich. They suggested that the star had just become a C-star, after HBB had ceased. The fact that it had the largest deviation from the $P L$-relation in the sample studied by Whitelock et al. (2003) seemed to support this hypothesis as this is also observed in O-rich stars. The much higher quality IRS spectrum shows no evidence for the presence of a silicate feature, as was originally discussed by Speck et al. (2006), who suggested that this apparent silicate emission is an artifact of the underestimation of the level of the continuum emission in the ISO spectrum. In fact, there is no evidence that this star is currently undergoing HBB.
In the Whitelock et al. sample it was the only C-star that lay significantly above the $P L$-relation, but one of the strengths of the present study is that it enlarges significantly the number of heavy mass-losing stars with a pulsation period. Figure 27 shows that IRAS 04496 still is the C-star that has the largest overluminosity w.r.t. classical $P L$-relations derived for shorter period miras, but no longer the only one. The relation proposed by Hughes \& Wood (1990) seems to delineate the upper boundary of luminosities that can be reached. For periods longer than $P=450$ days, where Hughes \& Wood proposed a break in the $P L$-relation, there is enormous scatter, which is not present in the $P L$-relation at shorter periods (the scatter found by Groenewegen \& Whitelock is $0.26 \mathrm{mag}$ ). This may be in part due to the fact that the luminosities in the present study are not from single-epoch photometry.

One peculiar object is MSX LMC 775, which is plotted at the period with the largest amplitude, 2063 days as derived from OGLE data, 2210 days as derived from MACHO red data. This is very unusual as the periods of C-stars are confined to $\lesssim 1000$ days (see Fig. 27; Whitelock et al. 2003; Kerschbaum et al. 2006; for Galactic C-stars), and this is consistent with the picture that HBB prevents the formation of $\mathrm{C}$-stars for large initial masses. This star could truly be an object that has turned into a C-star after HBB ceased. On the other hand this star also shows a significant period of 269 days with an amplitude of 0.46 in the $I$-band, also suggestive of mira pulsation. This would place the star in the 

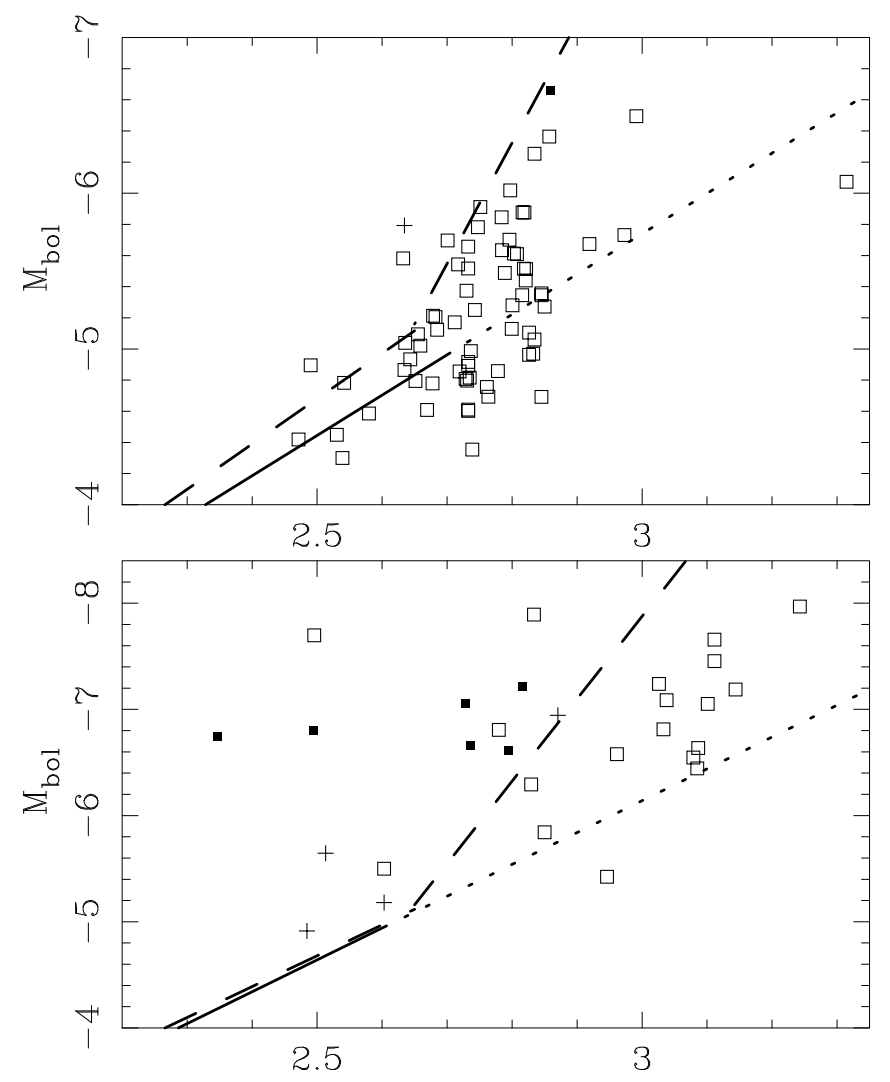

$\log$ Period

Fig. 27. As Fig. 26 but without the model tracks, without supergiants, and only variables with large amplitudes are plotted. Open squares indicate stars without information on the presence of HBB, plus-signs are stars without $\mathrm{HBB}$ (no Lithium detected), filled squares stars which undergo HBB (or supposed to, like the C-star IRAS 04496-6958, but which probably is not, see text).

upper left part in Fig. 27, also in a location not occupied by other $\mathrm{C}$-stars. From the MACHO data a third period of 549 days can be derived, which would place it among other C-stars, but the amplitude is smaller and unlike the pulsation of a Mira.

Regarding the O-rich Miras there are a few that follow the extrapolation of the classical $P L$-relation derived for shortperiod miras, but most are overluminous. Some are known to be rich in Lithium (the filled symbols) and it would be interesting to investigate this for all objects in Fig. 27 brighter than -6.0 in bolometric magnitude (although for some this will be difficult as they are very red).

\section{Summary and conclusions}

Mass-loss rates for the sample are derived under the assumption of a constant expansion velocity and dust-to-gas ratio with values similar to galactic AGB stars.

Wachter et al. (2008) compute dynamical wind models for subsolar metallicities for carbon stars. They find that the outflow velocities of the solar metallicity models are higher by about a factor of $2.2 \pm 0.2$ than those of the LMC, and $4 \pm 1$ than those of the SMC. The dust-to-gas ratios are larger by factors $1.3 \pm 0.1$ and $2.3 \pm 0.2$, respectively. For the modelling in this paper this means that the assumption of typical velocities and dust-to-gas ratios of galactic stars may lead to an overestimate of the mass-loss rates by a factor of $1.7 \pm 0.2$ for the LMC, and $1.7 \pm 0.5$ for the SMC targets, respectively (since the dust optical depth $\tau \sim \dot{M} \Psi / v_{\text {exp }}$ is fitted). From their Eqs. (1)-(3) for default parameter values $\left(M=1 M_{\odot}, T_{\text {eff }}=2600 \mathrm{~K}, L=10^{4} L_{\odot}\right)$, the ratio of mass-loss rates is $\mathrm{GAL}: \mathrm{LMC}: \mathrm{SMC}=1: 0.84: 0.52$.

Mattsson et al. (2009) computed a grid of 900 dynamical model atmospheres for carbon stars for solar metallicities spanning a range in luminosity, pulsation velocity amplitude, effective temperature, $(\mathrm{C}-\mathrm{O})$ excess and stellar mass. The online material includes output (mass-loss rates, expansion velocities and dust-to-gas ratios) for about 325 models. Taking only models with expansion velocities in the range $2-45 \mathrm{~km} \mathrm{~s}^{-1}$ only 253 "plausible" models remain. The $98 \%$ percentile on $\beta=$ $\left(\dot{M} v_{\text {exp }}\right) /(L / c)=3.0$. Given the scaling parameters as derived by Wachter et al. one would expect maximum values for $\beta$ a factor of 2.6 lower in the LMC and 7.7 lower in the SMC, respectively, than in our galaxy, or about 1.1 and 0.39 . From Table 3 the $98 \%$ percentile on $\beta=1.2$, while the largest value for an SMC Cstar is 0.52 . These values are consistent with predictions. The range in dust-to-gas ratio in the 253 models by Mattsson et al. is $(3-80) \times 10^{-4}$, which encompasses the adopted value of 0.005 .

At the level of a factor 2-4, the predicted and observed massloss rates agree and confirm that there is no strong dependence of mass-loss rate on metallicity. To have a better understanding of the mass-loss process it is crucial to measure the expansion velocity and determine the gas-to-dust ratio by observing the $\mathrm{CO}$ thermal emission lines in the (sub-)mm. In addition it would be valuable if the $\mathrm{C} / \mathrm{O}$ ratio could be determined for some stars in the sample, as the wind properties are predicted to depend on the excess of carbon over oxygen atoms.

In this last paragraph of the paper we want again to draw the attention to the pulsation properties of two remarkable objects (see Sect. 5.5). The C-star MSX LMX 775, which we classified as having a pulsation period of over 2000 days. This is much longer than the maximum period of all known Galactic and MC C-stars which is near 900 days. This period is located however on an extension of the C-star $P L$-relation derived for unobscured stars. If true, MSX LMC 775 could be a very massive star that has become a C-star after HBB ceased. The other remarkable object is the O-rich star MSX SMC 055 with a period of 1749 days and at $M_{\mathrm{bol}}=-8.0$. Its pulsation amplitude and colour are consistent with that of a (mass-losing) Mira, and not an RSG star. Its luminosity is consistent with that predicted for super-AGB stars, and therefore we propose this object as the most likely super-AGB star candidate from an observational point of view.

Acknowledgements. This publication is partly based on the OGLE observations obtained with the Warsaw Telescope at the Las Campanas Observatory, Chile, operated by the Carnegie Institution of Washington.

This paper utilizes public domain data originally obtained by the MACHO Project, whose work was performed under the joint auspices of the US Department of Energy, National Nuclear Security Administration by the University of California, Lawrence Livermore National Laboratory under contract No. W-7405-Eng-48, the National Science Foundation through the Center for Particle Astrophysics of the University of California under cooperative agreement AST-8809616, and the Mount Stromlo and Siding Spring Observatory, part of the Australian National University.

This research has made use of the SIMBAD database, operated at CDS, Strasbourg, France.

The authors would like to thank Dr. Mikako Matsuura for making the lists of Oand C-stars discussed in Matsuura et al. (2009) available.

M.G. would like to thanks Ariane Lançon and Eric Josselin for organising the "Intermediate Mass Stars $\leftrightarrow$ Massive Stars, A workshop around causes and consequences of differing evolutionary paths" meeting in Strasbourg, which was inspiring and resulted in some ideas being explored in this paper. 
Table A.1. Multi-period information.

\begin{tabular}{|c|c|c|c|c|c|c|c|}
\hline Identifier & Period & Amp. & Period & Amp. & Period & Amp. & Remarks \\
\hline MSX LMC 587 & $644 \pm 2$ & 0.32 & $340 \pm 1.3$ & 0.18 & & & ASAS I \\
\hline HD 271832 & $514 \pm 1.1$ & 0.16 & $52.66 \pm 0.02$ & 0.057 & $142.0 \pm 0.2$ & 0.060 & ASAS V \\
\hline WBP 51 & $382.06 \pm 0.04$ & 0.11 & $439.80 \pm 0.07$ & 0.09 & $258.36 \pm 0.06$ & 0.03 & MACHO R \\
\hline MSX LMC 791 & $436.31 \pm 0.02$ & 0.17 & $1654.1 \pm 0.2$ & 0.19 & & & MACHO B \\
\hline MSX LMC 1318 & $683.36 \pm 0.07$ & 0.14 & $303.94 \pm 0.02$ & 0.11 & & & MACHO R \\
\hline WOH S 264 & $453.44 \pm 0.04$ & 0.10 & $2830 \pm 1$ & 0.29 & & & MACHO B \\
\hline MSX LMC 1492 & $559.00 \pm 0.04$ & 0.71 & $2309.4 \pm 0.4$ & 1.34 & & & MACHO R \\
\hline MSX LMC 1492 & $564.29 \pm 0.12$ & 0.59 & $2545.1 \pm 1.4$ & 1.06 & $276.24 \pm 0.04$ & 0.30 & OGLE I \\
\hline MSX LMC 218 & $670.5 \pm 0.2$ & 0.52 & $2204 \pm 4$ & 0.26 & & & MACHO R \\
\hline MSX LMC 218 & $662.8 \pm 0.2$ & 0.74 & $3008 \pm 7$ & 0.36 & & & OGLE I \\
\hline MSX LMC 775 & $2209.1 \pm 0.85$ & 0.67 & $281.99 \pm 0.03$ & 0.34 & $548.7 \pm 0.13$ & 0.27 & MACHO R \\
\hline MSX LMC 775 & $2063 \pm 2.3$ & 1.83 & $269.46 \pm 0.05$ & 0.46 & & & OGLE I \\
\hline WOH G 64 & $855.79 \pm 0.03$ & 0.68 & $2647 \pm 1.0$ & 0.26 & & & MACHO R \\
\hline HV 11366 & $182.787 \pm 0.003$ & 0.32 & $292.02 \pm 0.02$ & 0.14 & & & MACHO B \\
\hline MSX LMC 768 & $626.7 \pm 0.5$ & 0.91 & $3501 \pm 27$ & 0.64 & $317.6 \pm 0.7$ & 0.15 & OGLE I \\
\hline MSX LMC 1282 & $655.1 \pm 0.58$ & 0.94 & $5503 \pm 68$ & 1.80 & & & OGLE I \\
\hline MSX LMC 937 & $658.9 \pm 1.0$ & 0.69 & $1515 \pm 26$ & 0.13 & & & OGLE I \\
\hline MSX LMC 1205 & $558.5 \pm 0.2$ & 0.94 & $3015 \pm 7$ & 1.20 & & & OGLE I \\
\hline MSX LMC 663 & $422.0 \pm 0.5$ & 0.02 & $3520 \pm 18$ & 0.05 & & & OGLE I \\
\hline MSX LMC 220 & $624.99 \pm 0.08$ & 0.58 & $2305.3 \pm 0.9$ & 0.62 & & & OGLE I \\
\hline MSX LMC 1308 & $501.9 \pm 0.2$ & 0.71 & $3430 \pm 22$ & 4.22 & & & OGLE I \\
\hline MSX LMC 95 & $609.06 \pm 0.10$ & 1.05 & $1664 \pm 2$ & 0.28 & & & OGLE I \\
\hline MSX LMC 1120 & $635.28 \pm 0.21$ & 0.89 & $1835 \pm 2$ & 0.79 & & & OGLE I \\
\hline MSX SMC 066 & $521.00 \pm 0.05$ & 0.36 & $1837.3 \pm 0.2$ & 0.96 & & & OGLE I \\
\hline MSX LMC 438 & $615.0 \pm 0.6$ & 0.64 & $1864 \pm 2$ & 1.24 & & & OGLE I \\
\hline MSX SMC 014 & $317.0 \pm 0.7$ & 1.17 & $2811 \pm 56$ & 1.56 & & & OGLE I \\
\hline NGC 419 IR1 & $476.97 \pm 0.04$ & 0.77 & $4778 \pm 5$ & 0.59 & & & OGLE I \\
\hline MSX LMC 783 & $515.1 \pm 0.2$ & 0.85 & $2206 \pm 10$ & 0.35 & & & OGLE I \\
\hline MSX LMC 634 & $484.42 \pm 0.10$ & 0.77 & $1463.3 \pm 1.1$ & 0.51 & & & OGLE I \\
\hline ISO 00549 & $683.3 \pm 0.2$ & 0.56 & $3029.2 \pm 1.4$ & 1.10 & & & OGLE I \\
\hline ISO 00548 & $432.54 \pm 0.03$ & 1.11 & $1345.8 \pm 0.4$ & 0.42 & & & OGLE I \\
\hline MSX SMC 093 & $457.82 \pm 0.18$ & 0.28 & $3159 \pm 3$ & 0.97 & & & OGLE I \\
\hline MSX LMC 754 & $448.16 \pm 0.10$ & 0.71 & $2303 \pm 10$ & 0.23 & & & OGLE I \\
\hline MSX SMC 232 & $466.73 \pm 0.13$ & 0.75 & $2452 \pm 2$ & 1.44 & & & OGLE I \\
\hline NGC 419 LE16 & $423.25 \pm 0.04$ & 0.29 & $1882.4 \pm 0.3$ & 0.64 & & & OGLE I \\
\hline MSX SMC 055 & $1749.1 \pm 0.2$ & 0.87 & $901.9 \pm 0.2$ & 0.24 & & & OGLE I \\
\hline IRAS 04509-6922 & $1240.70 \pm 0.11$ & 1.50 & $659.32 \pm 0.11$ & 0.41 & & & OGLE I \\
\hline IRAS 04516-6902 & $1084.17 \pm 0.14$ & 1.32 & $5436 \pm 31$ & 0.65 & & & OGLE I \\
\hline MSX LMC 642 & $1122.46 \pm 0.13$ & 1.65 & $552.1 \pm 0.2$ & 0.24 & & & OGLE I \\
\hline IRAS 05558-7000 & $1176.6 \pm 0.3$ & 2.34 & $556.5 \pm 0.3$ & 0.34 & & & OGLE I \\
\hline MSX SMC 024 & $417.97 \pm 0.11$ & 0.12 & $227.40 \pm 0.05$ & 0.08 & & & OGLE I \\
\hline IRAS 05003-6712 & $909.38 \pm 0.11$ & 1.39 & $446.68 \pm 0.14$ & 0.29 & & & OGLE I \\
\hline MSX SMC 134 & $247.90 \pm 0.12$ & 0.042 & $140.75 \pm 0.04$ & 0.04 & & & OGLE I \\
\hline WBP 77 & $215.21 \pm 0.01$ & 0.37 & $108.025 \pm 0.008$ & 0.12 & & & OGLE I \\
\hline
\end{tabular}

\section{Appendix A: Multi-periodic AGB stars}

Some of the stars for which we (re-)analysed ASAS, MACHO and OGLE data show multiple periods. Tables 1 and 2 list the (adopted) pulsation period. In the table below all derived periods are given The first period is the adopted pulsation period, then the other periods are listed. Typically only one additional period is fitted, because of the irregular behaviour. Only in exceptional cases a third period is fitted. Formal errors in the periods are also given. In the case of MACHO data, the blue channel was analysed only if the red channel data was corrupt.

There are 41 unique stars listed in the table. The second period fitted is in the range $0.4-0.6$ times the primary period in 10 cases. The amplitude of the second period is in the range $0.1-1.0$ times the amplitude of the primary period. These cases probably represent the situation where a pulsation mode and the next higher overtone mode are detected. When there is an LSP, it is mostly in the range $3-10$ times the primary period.

\section{References}

Begemann, B., Dorschner, J., Henning, Th., et al. 1997, ApJ, 476, 199 Bertelli, G., Girardi, L., Marigo, P., \& Nasi, E. 2008, A\&A, 484, 815 Blommaert, J. A. D. L., Groenewegen, M. A. T., Okumura, K., et al. 2006, A\&A, 460,555

Bolatto, A. D., Simon, J. D., Stanimirović, S., et al. 2007, ApJ, 655, 212 Borghesi, A., Bussoletti, E., Colangeli, L., \& de Blasi, C. 1985, A\&A, 153, 1

Buchanan, C. L., Kastner, J. H., Forrest, W. J., et al. 2006, AJ, 132, 1890

Cioni M.-R., Loup C., Habing H. J., et al. 2000, A\&AS, 144, 235

Cioni, M.-R. L., Blommaert, J. A. D. L., Groenewegen, M. A. T., et al. 2003, A\&A, 406, 51

David, P., \& Pégourié, B. 1995, A\&A, 293, 833

DENIS Consortium 2005, available via ViZier

DePew, K., Speck, A., \& Dijkstra, C. 2006, ApJ, 640, 971

Dodion, J. 2003, M.Sc. Thesis, University of Leuven

Dorschner, J., Begemann, B., Henning, T., Jaeger, C., \& Mutschke, H. 1995, A\&A, 300, 503

Draine, B. T., \& Lee, H. M. 1984, ApJ, 285, 89

Feast, M. W., Glass, I. S., Whitelock, P. A., \& Catchpole, R. M. 1989, MNRAS, 241,375

Fluks, M. A., Plez, B., Thé, P. S., et al. 1994, A\&AS, 105, 311

Fraser, O., Hawley, S. L, \& Cook, K. 2008, AJ, 136, 1242 
Goebel, J. H., \& Moseley, S. H. 1985, ApJ, 290, L35

Groenewegen, M. A. T. 1993, Ph.D. Thesis, Chapter 5, University of Amsterdam Groenewegen, M. A. T. 1995, A\&A, 293, 463

Groenewegen, M. A. T. 2004, A\&A, 425, 595

Groenewegen, M. A. T. 2006, A\&A, 448, 181

Groenewegen, M. A. T., \& Blommaert, J. A. D. L. 2005, A\&A, 443, 143

Groenewegen, M. A. T., \& de Jong, T. 1993, A\&A, 267, 410

Groenewegen, M. A. T., \& Whitelock, P. A., 1996, MNRAS, 281, 1347

Groenewegen, M. A. T., Whitelock, P. A., Smith, C. H., \& Kerschbaum F. 1998, MNRAS, 293, 18

Groenewegen, M. A. T., Wood, P. R., Sloan, G. C., et al. 2007, MNRAS, 367, 313

Harwit, M., Malfait, K., Decin, L., et al. 2001, ApJ, 557, 844

Heras, A. M., \& Hony, S. 2005, A\&A, 439, 171

Höfner, S. 2008, A\&A, 491, L1

Hony, S., Waters, L. B. F. M., \& Tielens, A. G. G. M. 2002, A\&A, 390, 533

Houck J. R., Roelling T. L., van Cleve J., et al. 2004, ApJS, 154, 18

Hughes, S. M. G. 1989, AJ, 97, 1634

Hughes, S. M. G., \& Wood, P. R. 1990, AJ,99, 784

Izzard, R. G., Tout, C. A., Karakas, A. I., \& Pols, O. R. 2004, MNRAS, 350, 407

Jones, T. W., \& Merrill, K. M. 1976, ApJ, 209, 509

Jørgensen, U. G., Hron, J., \& Loidl, R. 2000, A\&A, 356, 253

Kastner, J. H., Thorndike, S. L., Romanczyk, P. A., et al. 2008, AJ 136, 122

Kato, D., Nagashima, C., Nagayama, T., et al. 2007, PASJ, 59, 615

Kemper, F., de Koter, A., Waters, L. B. F. M., Bouwman, J., \& Tielens, A. G. G. M. 2002, A\&A, 384, 585

Kerschbaum, F., Groenewegen, M. A. T., \& Lazaro, C. 2006, A\&A, 460, 539

Kozasa, T., Dorschner, J., Henning, T., \& Stognienko, R. 1996, A\&A, 307, 551

Kraemer, K. E., Sloan, G. C., Wood, P. R., Price, S. D. \& Egan, M. P. 2005, ApJ, 631, L147

Kraemer, K. E., Sloan, G. C., Bernard-Salas, J., et al. 2006, ApJ, 652, L25

Lagadec, E., Zijlstra, A. A., Sloan, G. C., et al., 2007, MNRAS, 376, 1270

Lebzelter, T., \& Wood, P. R. 2005, A\&A, 441, 1117

Lebzelter, T., \& Wood, P. R. 2007, A\&A, 475, 643

Leisenring, J. M., Kemper, F., \& Sloan, G. C. 2008, ApJ, 681, 1557

Levesque, E. M., Massey, P., Olsen, K. A. G., et al. 2005, AJ, 628, 973

Levesque, E. M., Massey, P., Olsen, K. A. G., et al. 2006, AJ, 645, 1102

Loidl R., Lançon A., \& Jrgensen U. G. 2001, A\&A, 371, 1065

Lloyd Evans, T. 1985, MNRAS, 212, 955

Martin, P. G., \& Rogers, C. 1987, ApJ, 322, 374

Massey, P. 2002, ApJS, 141, 81

Matsuura, M., Wood, P. R., Sloan G. C., et al., 2006, MNRAS, 371, 415

Matsuura, M., Barlow, M. J., Zijlstra, A. A., et al. 2009, MNRAS, accepted

Mattsson, L., Wahlin, R., Höfner, S., \& Eriksson, K. 2008, A\&A, 484, L5

Mattsson, L., Wahlin, R., \& Höfner, S. 2009, A\&A, submitted

Meixner, M., Gordon, K. D., Indebetouw, R., et al. 2006, AJ, 132, 2268

Min, M., Hovenier, J. W., \& de Koter, A. 2003, A\&A, 404, 35

Min, M., Hovenier, J. W., \& de Koter, A. 2005, A\&A, 432, 909

Min, M., Waters, L. B. F. M., de Koter, A., et al. 2007, A\&A, 462, 667

Moshir, M., Copan, G., Conrow, T., et al. 1989, The IRAS Faint Source Catalog, version 2

Nishida, S., Tanabé, T., Nakada, Y., et al. 2000, MNRAS, 313, 136

Oestreicher M. O., Schmidt-Kaler T., \& Wargau W. 1997, MNRAS, 289, 729

Ohnaka, K., Driebe, T., Hofmann, K.-H., Weigelt, G., \& Wittkowski, M. 2008, A\&A, 484, 371

Ordal, M. A., Bell, R. J., Alexander, R. W., Jr., Newquist, L. A., \& Querry, M. R. 1988, ApOpt, 27, 1203

Ossenkopf, V., Henning, Th., \& Mathis, J. S. 1992, A\&A, 261, 567
Paczyński B. 1970, AcA, 20, 47

Papoular, R. 2008, MNRAS, 388, 457

Payne-Gaposchkin, C. H. 1971, Smithsonian Contr. Ap., 13, 1

Pégourié B. 1988, A\&A, 194, 335

Pitman, K. M., Hofmeister, A. M., Corman, A. B., \& Speck, A. K. 2008, A\&A, 483, 661

Poelarends, A. J. T., Herwig, F., Langer, N., \& Heger, A. 2008, ApJ, 675, 614 Pojmanski, G. 2002, AcA, 52,397

Posch, T., Kerschbaum, F., Mutschke, H., et al. 1999, A\&A, 352, 609

Press, W. H., Teukolsky, S. A., Vetterling, W. T., \& Flannery, B. P. 1992, in

Numerical Recipes in Fortran (Cambridge U. P.), 77

Raimondo, G., Cioni, M.-R. L., Rejkuba, M., \& Silva, D. R. 2005, A\&A, 438, 521

Reid, N., Glass, I. S., \& Catchpole, R. M. 1988, MNRAS, 232, 53

Rouleau, F., \& Martin, P. G., 1991, ApJ, 377, 526

Schwarz, G. 1978, Ann. Stat., 6, 461

Skrutskie M. F., Cutri, R. M., Stiening, R., et al. 2006, AJ, 131, 1163

Sloan, G. C., \& Price, S. D. 1995, ApJ, 451, 758

Sloan, G. C., LeVan, P. D., \& Little-Marenin, I. R. 1996, ApJ, 463, 310

Sloan, G. C., Kraemer, K. E., Goebel, J. H., \& Price, S. D. 2003, ApJ, 594, 483

Sloan, G. C., Kraemer, K. E., Matsuura, M. 2006, ApJ, 645, 1118

Sloan, G. C., Kraemer, K. E., Wood, P. R., et al. 2008, ApJ, 686, 1056

Sloan, G. C., Matsuura, M., Zijlstra, A. A., et al. 2009, Science 323, 353

Smith, V. V., \& Lambert, D. L. 1989, ApJ, 345, L75

Smith, V. V., \& Lambert, D. L. 1990, ApJ, 361, L69

Smith, V. V., Plez, B., Lambert, D. L., \& Lubowich, D. A. 1995, ApJ, 441, 735

Soszyński, I. 2007, ApJ, 660, 1486

Speck, A. K., Cami, J, Marwick-Kemper, C., et al. 2006, ApJ, 650, 892

Speck, A. K., Corman, A. B., Wakeman K., Wheeler, C. H., \& Thompson, G. 2009, ApJ, 691, 1202

Suh, K.-W. 1999, MNRAS, 304, 389

Treffers, R., \& Cohen, M. 1974, ApJ, 188, 545

Trams, N. R., van Loon, J.Th., Zijlstra, A. A., et al. 1999, A\&A, 344, L17

Udalski, A., Szymanski, M., \& Kubiak, M. 1998, AcA, 48, 147

Udalski, A., Szymanski, M. K., Soszynski, I., \& Poleski, R. 2008, AcA 58, 69

Vassiliadis, E, \& Wood, P. R. 1993, ApJ, 413, 641

Verhoelst, T., Van der Zypen, N., Hony, S., et al. 2009, A\&A, 498, 127

Vijh, U. P., Meixner, M., Babler, B., et al. 2009, AJ, 137, 3139

Volk, K., \& Kwok, S. 1988, ApJ, 331, 435

Volk, K., Kwok, S., \& Langill, P. P. 1992, ApJ, 391, 285

Wachter, A., Winters, J. M., Schröder, K.-P., \& Sedlmayr, E. 2008, A\&A, 486,

Wagenhuber, J., \& Groenewegen, M. A. T. 1998, A\&A, 340, 183

Werner M. W., Roellig T. L., Low F. J., et al. 2004, ApJS, 154, 1

Whitelock, P., Menzies, J., Feast, M., et al. 1994, MNRAS, 267, 711

Whitelock P. A., Feast M. W., Menzies J. W., \& Catchpole R. M., 1989, MNRAS, 238, 769

Whitelock, P. A., Feast, M. W., van Loon, J.Th., \& Zijlstra, A. A. 2003, MNRAS, 342,86

Wood, P. R. 1998, A\&A, 338, 592

Wood, P. R., \& Zarro, D. M. 1981, ApJ, 247, 247

Wood, P. R., Bessell, M. S., \& Fox, M. W. 1983, ApJ, 27299 (WBF)

Wood, P. R., Whiteoak, J. B., Hughes, S. M. G., et al. 1992, ApJ, 397, 552

Zaritsky D., Harris J., Thompson I. B., Grebel E. K., \& Massey P. 2002, AJ, 123, 855

Zaritsky D., Harris J., Thompson I. B., \& Grebel E. K. 2004, AJ, 128, 1606 Zijlstra, A. A., Matsuura, M., Wood, P. R., et al., 2006, MNRAS, 370, 1961 Zhukovska, S., \& Gail, H.-P. 2008, A\&A, 486, 229 
M. A. T. Groenewegen et al.: Luminosities and mass-loss rates of SMC and LMC AGB stars and red supergiants, Online Material $p 1$
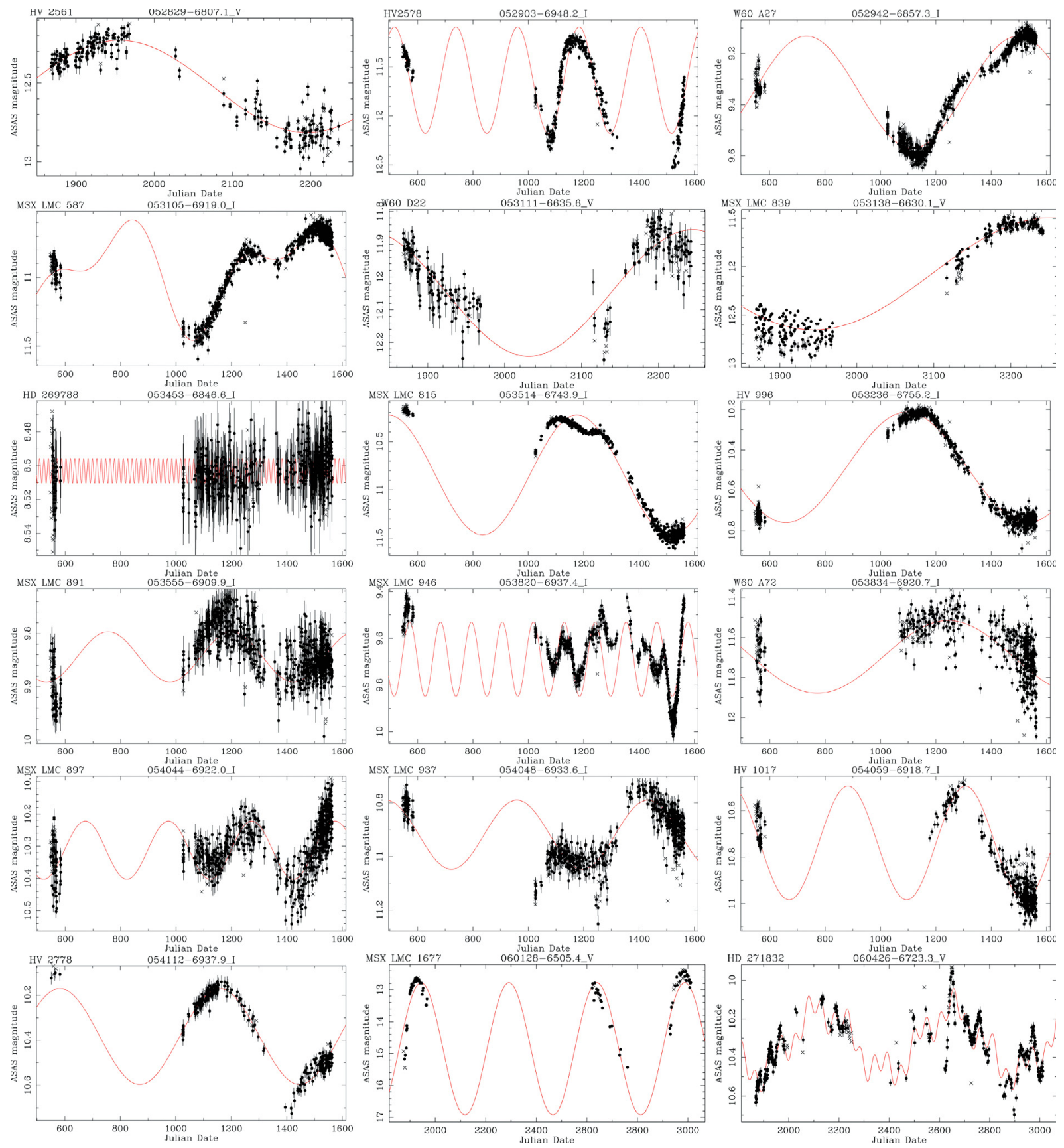

Fig. 1. Sample lightcurves and fits to ASAS data. The identifier used in the present paper and the ASAS identifier are listed on top of the plot. Julian Date plotted is JD-2 450000. 
M. A. T. Groenewegen et al.: Luminosities and mass-loss rates of SMC and LMC AGB stars and red supergiants, Online Material p 2
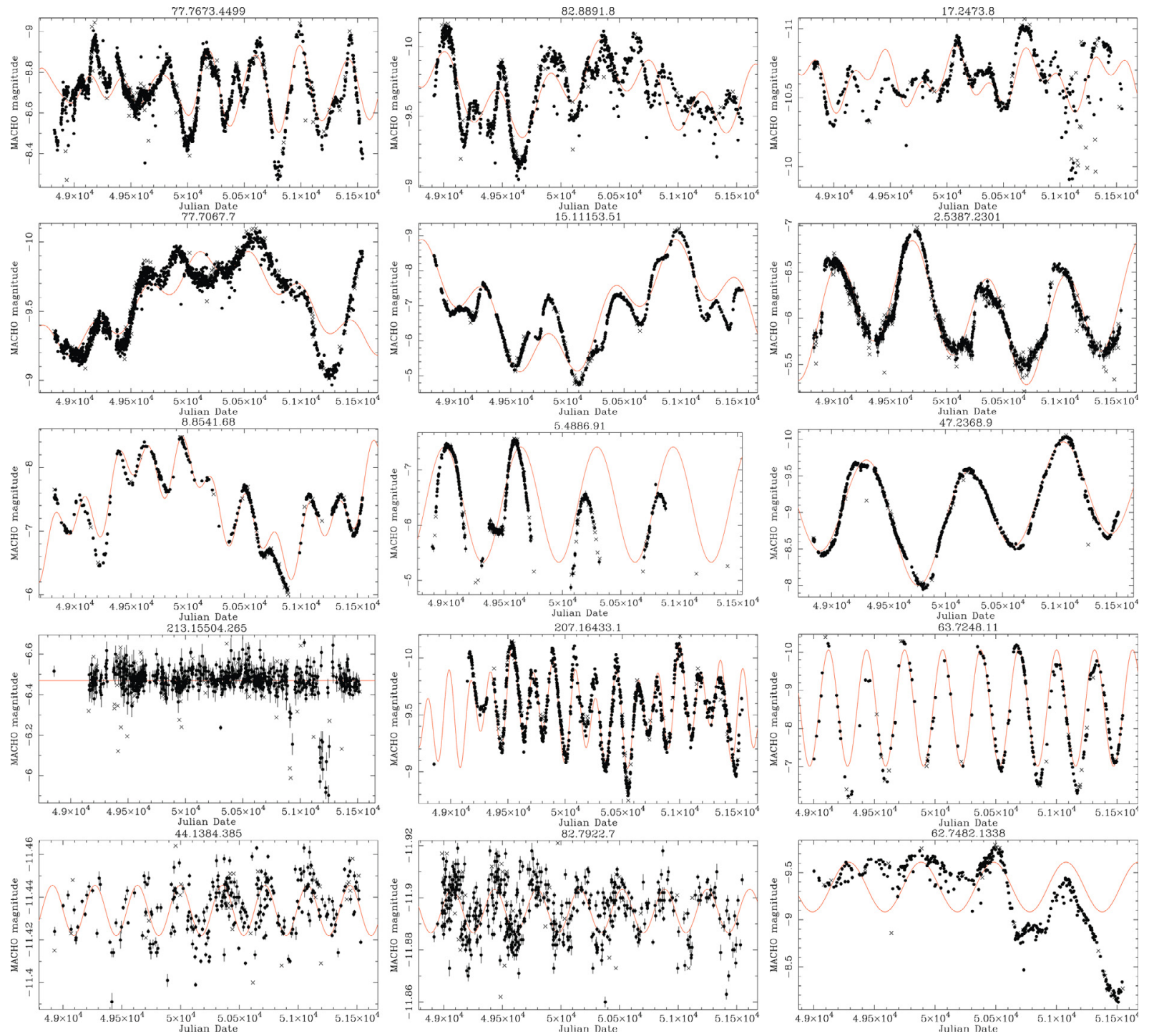

Fig. 2. Sample lightcurves and fits to MACHO data. MACHO identifiers are listed on top of the plot, and are cross-referenced in Tables 1 and 2. Julian Date plotted is JD-2 400000. 
M. A. T. Groenewegen et al.: Luminosities and mass-loss rates of SMC and LMC AGB stars and red supergiants, Online Material p 3
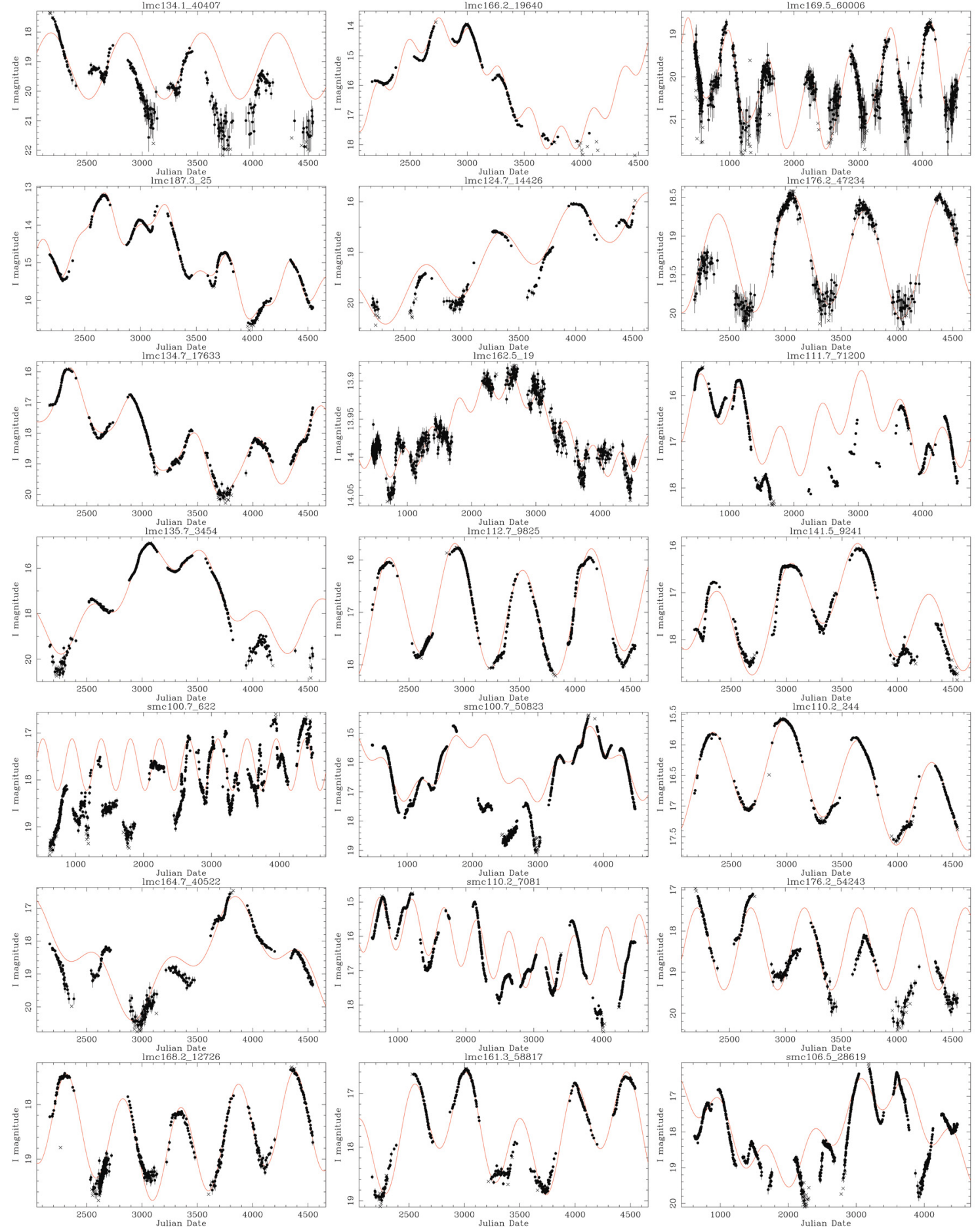

Fig. 3. Sample lightcurves and fits to OGLE-III data (and OGLE-II data when available). OGLE-III identifiers are listed on top of the plot, and are cross-referenced in Tables 1 and 2. Julian Date plotted is JD-2 450000. 
M. A. T. Groenewegen et al.: Luminosities and mass-loss rates of SMC and LMC AGB stars and red supergiants, Online Material p 4
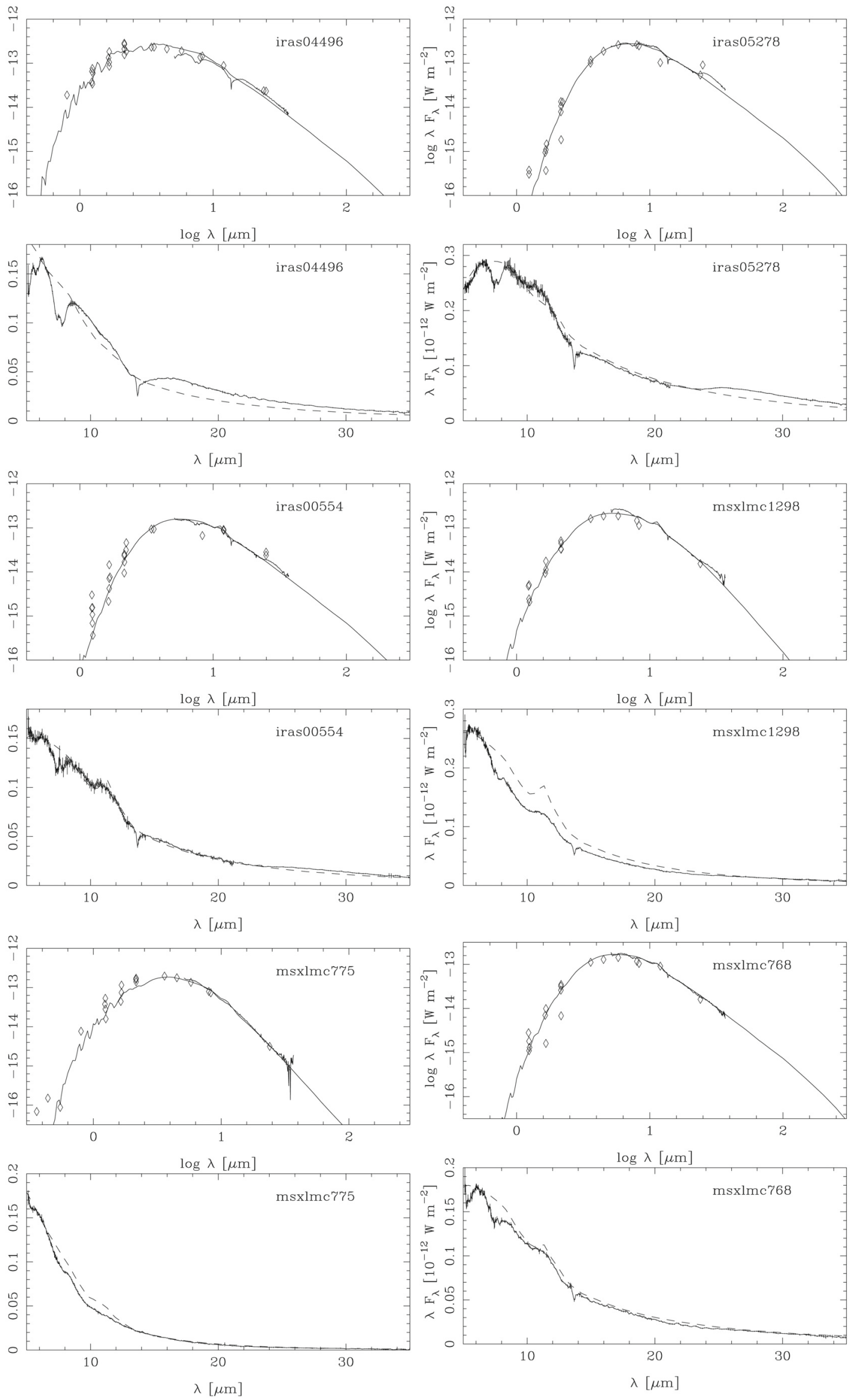

Fig. 5. Fits to the SEDs and IRS spectra of C-stars. 
M. A. T. Groenewegen et al.: Luminosities and mass-loss rates of SMC and LMC AGB stars and red supergiants, Online Material p 5
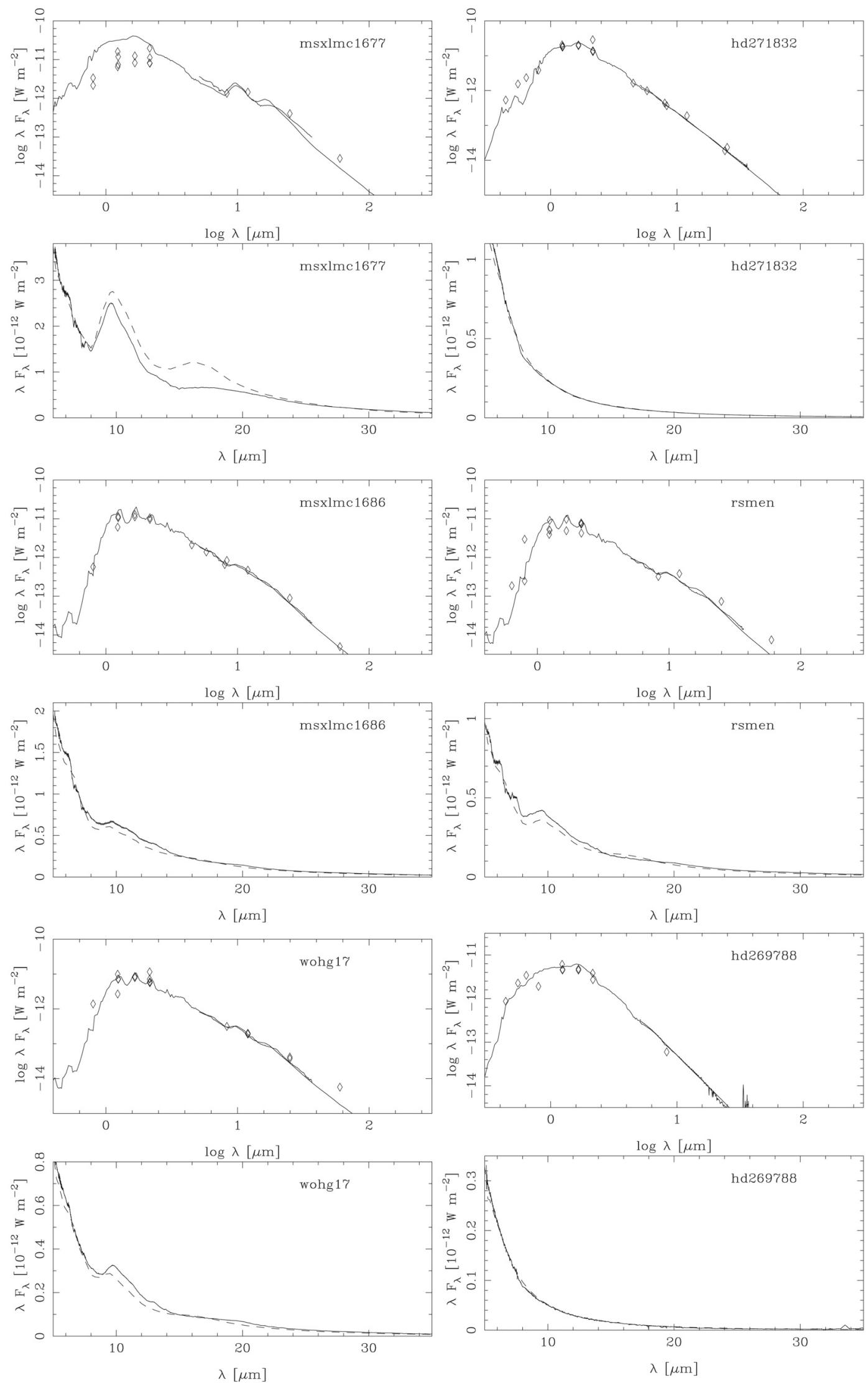

Fig. 6. Fits to the SEDs and IRS spectra of M-stars using "laboratory silicates". 
M. A. T. Groenewegen et al.: Luminosities and mass-loss rates of SMC and LMC AGB stars and red supergiants, Online Material p 6
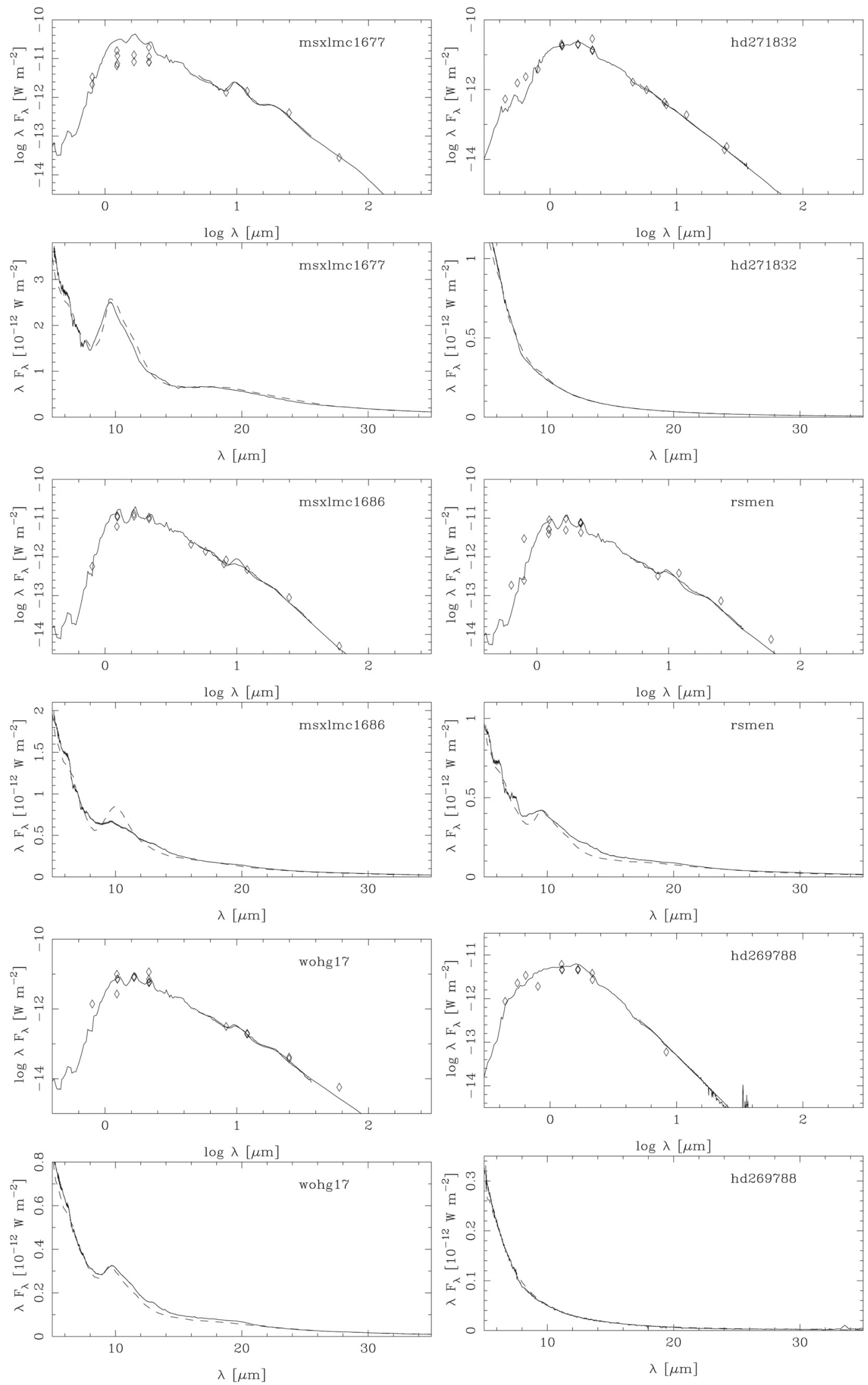

Fig. 7. Fits to the SEDs and IRS spectra of M-stars using "astronomical silicates". 